\title{
Hippocampal Contributions to Neurocognitive Mapping in Humans: A New Model
}

\author{
R.A.P. Roche, ${ }^{1,2}$ M.A. Mangaoang, ${ }^{2}$ S. Commins, ${ }^{1}$ and S.M. O'Mara ${ }^{2 *}$
}

\begin{abstract}
The ability of an organism to develop, maintain, and act upon an abstracted internal representation of spatially extensive environments can provide an increased chance in ensuring that organism's survival. Here, we propose a neurocognitive model of spatial representation describing how several different processes interact and segregate the differing types of information used to produce a unified cognitive map. This model proposes that view-based egocentric and vestibulomotor translational information are functionally and anatomically separate, and that these parallel systems result in independent, but interacting, models within a neurocognitive map of space. In this context, we selectively review relevant portions of the large literature, addressing the establishment and operation of such spatial constructs in humans and the brain systems that underpin them, with particular reference to the hippocampal formation (HF). We present a reinterpretation of the types of knowledge used in the formation of this spatial construct, the processes that act upon this information, the nature of the final spatial representation, and describe how these universal concepts relate to the proposed model of spatial processing. The relevant experimental paradigms used to examine the neural basis of spatial representation and the main findings from previous research are also briefly presented. Finally, we detail a series of testable theoretical, behavioral, and anatomical predictions made by the model. $\odot 2005$ Wiley-Liss, Inc.
\end{abstract}

KEY WORDS: spatial representation; spatial navigation; humans; hippocampal formation; path integration

\section{INTRODUCTION}

A prerequisite for the survival of a behaving organism is its ability to interact effectively and efficiently with its environment (Kamil, 1994; Shettleworth, 1998). Tasks such as foraging or hunting for food, locating shelter, avoiding predators, and remembering dangerous locations all require a reliable system whereby important elements of the external world, and their relations to each other and to the organism, are repre-

\footnotetext{
${ }^{1}$ Department of Psychology, National University of Ireland, Maynooth, County Kildare, Ireland; ${ }^{2}$ Department of Psychology and Trinity College Institute of Neuroscience, University of Dublin, Trinity College, Dublin, Ireland

M.A. Mangaoang is currently at St. Patrick's Hospital, P.O. Box 136, James's St., Dublin 8, Ireland.

Grant sponsor: Higher Education Authority Research (HEA; Programme for Research in Third Level Institutions [PRTLI]).

*Correspondence to: S.M. O'Mara, Department of Psychology and Trinity College Institute of Neuroscience, University of Dublin, Trinity College, Dublin 2, Ireland. E-mail: smomara@tcd.ie

Accepted for publication 8 March 2005

DOI 10.1002/hipo.20084

Published online 15 June 2005 in Wiley InterScience (www.interscience. wiley.com).
}

sented (Healy, 1998; Jacobs, 1995). This internal model of the environment has been termed either a spatial representation or a cognitive map, and the behavior of negotiating a route through the world based on this model is spatial navigation. The task of understanding the development of these capacities and their neuroanatomical underpinnings has occupied neuroscience research for several decades, and a large literature now exists on the topic in rodents (e.g., Redish, 1999; Thinus-Blanc et al., 1998; Schenk, 1998; Mumby et al., 1999), primates (e.g., Rolls, 1996; Rolls and O’Mara, 1995; O’Mara et al., 1994; Rolls et al., 1989a, 1989b; Feigenbaum and Rolls, 1991) and humans (e.g., Berthoz, 1999, 1997; Berthoz et al., 1995; Ghaem et al., 1997; Maguire et al., 1999). In the present study, we propose a new and simple predictive model of how spatial representations are generated by humans.

To construct an adequate cognitive representation of the external world containing information necessary for successful navigation, a brain structure(s) will require information from different sensory modalities. These will include visual input from occipital cortex, auditory information from temporal association areas, balance and topographical information from the vestibular system, and proprioceptive feedback about muscles and joints from somatosensory areas, as well as (to a lesser extent in humans) olfactory information from the olfactory bulb. There are only two strong candidate areas that have access to the requisite sensory stimulation necessary to develop a robust representation of the animal's place in the environment: the hippocampal formation of the medial temporal lobe (MTL) and, to a lesser extent, the parietal cortex. In recent years, lesion studies in humans and animals, as well as electrophysiological and neuroimaging studies, have confirmed the involvement of both these areas in spatial representation. This paper focuses particularly on the role of the hippocampal formation (HF) and, to a lesser extent, the parietal cortex, in spatial representation and navigation. In the present work, we do not attempt an analysis of the relationship between hippocampal formation function and amnesia, as many reviews of this topic have been published in recent years (e.g., Spiers et al., 2001; Dash et al., 2004; Mayes and Montaldi, 2001). 


\section{ANATOMY OF THE MEDIAL TEMPORAL LOBE}

The hippocampal formation is a bilateral, archicortical structure in the medial temporal lobe consisting of the hippocampus proper (also known as Ammon's horn or cornu ammonis (CA1CA3)), 63+dentate gyrus, and subiculum. Bordering the HF proper are the entorhinal, perirhinal, and parahippocampal cortices that surround the rhinal sulcus on the ventromedial surface of the primate brain (Lavenex and Amaral, 2000).

Sensory information accesses the HF through direct and indirect projections from perirhinal, parahippocampal areas (Suzuki and Amaral, 1994a,b; Naber et al., 1997, 1999), and medial and lateral entorhinal cortices (Witter et al., 1989; Witter and Groenewegen, 1992; Lopes da Silva et al., 1990; Leung et al., 1995), as well as prefrontal cortex. Although dentate gyrus and area CA3 do not seem to receive direct projections from either perirhinal or parahippocampal cortices (Canning and Leung, 1997; Naber et al., 1999), both areas receive indirect sensory information via cells in layer II of the entorhinal cortex along the perforant pathway. The dentate gyrus projects directly to area CA3 via mossy fibers. In turn a series of fibers termed the Schaffer collaterals send information from area CA3 to area CA1 (see Fig. 1A). Area CA1 then projects back to entorhinal and perirhinal cortices directly or indirectly via the subiculum. The entorhinal, perirhinal and parahippocampal cortices serve not only as the major ports of entry into the HF but also as its major cortical output via dense, reciprocal projections (see Fig. 1B). Thus, the perirhinal and parahippocampal cortices constitute the major communication links between widespread areas of motor and sensory association cortex on the one hand and the HF on the other.

In addition, the HF has extensive feedback projections to the cerebral cortex arising from subicular and CA1 neurons that terminate throughout the orbitofrontal, medial frontal, anterior temporal, and posterior temporal association cortices. Considered together, these projections serve powerful hippocampo-cortical neural systems influencing both the limbic and association cortices (Van Hoesen, 1995). Subcortical outputs occur primarily through the fimbria and include projections to the septal nuclei, nucleus accumbens, thalamus, and mammillary nuclei. There are also extensive commissural connections between left and right HF.

\section{Role of the Hippocampal Formation in Spatial Representation and Navigation}

Its distinctive anatomy is probably responsible to some degree for the remarkable level of interest in the function of the HF; furthermore, three key experimental observations have been the main driving forces behind the plethora of investigations, theories and debates on the function of this structure. The discovery of place cells in the rodent by O'Keefe and Dostrovsky (1971) was a landmark achievement. Similarly, the findings from clinical neuropsychological cases that lesions of the HF can cause profound anterograde amnesia (Scoville and
A

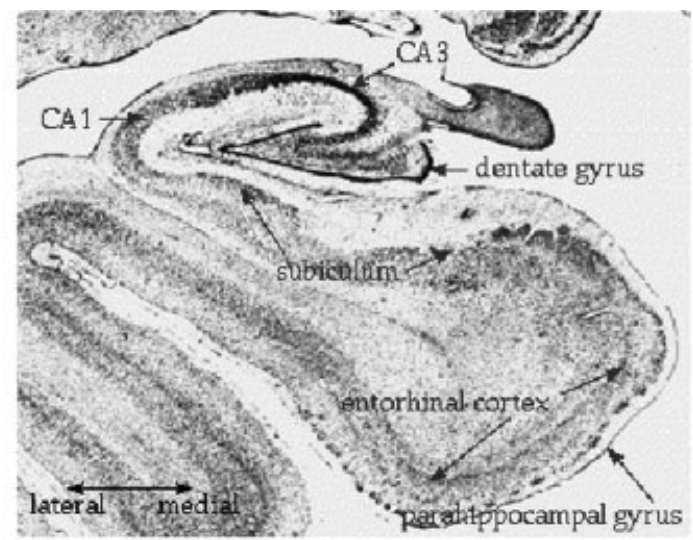

B

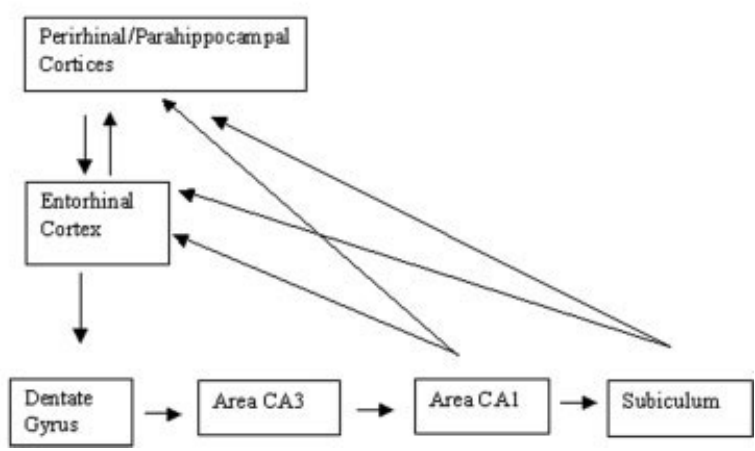

FIGURE 1. A: Line drawing of a cross section through the hippocampal formation illustrating the major areas of the hippocampal formation (HF). (Image from http://thalamus.wustl.edu/ course/limbic.html.) B: Flow diagram summarizing the intrinsic connections of the HF and also the projections to and from the parahippocampal and perirhinal cortices.

Milner, 1957) provided a strong impetus to increase our understanding of the functional role played by the HF. Finally, longterm potentiation (LTP), the neurophysiological process whereby connectivity between cells is altered due to experience, was first demonstrated in the $\mathrm{HF}$ and this area remains the region of brain in which it is still most investigated (Bliss and Lømo, 1973). There are many theories and models of hippocampal functioning (e.g., Rolls, 1996; Squire, 1992; Cohen and Eichenbaum, 1993), the most famous of which is the Cognitive Map Theory proposed by O'Keefe and Nadel (1978), which will be discussed below.

\section{DEVELOPING A REPRESENTATION OF SPACE}

The development of a mental model of the spatial world begins with sensory input from the environment. The processes whereby these sensory inputs are translated into a stored representation or model of the environment have been referred to as spatial representations or spatial strategies; we will use the term "spatial strategies" to describe the processes which correspond to the encoding aspect of spatial memory formation. We do so 
for two reasons. First, the term "strategies" conveys the dynamic nature of the processes involved. Second, the term "representations" can be misleading in that it implies a fixed or static model of space, rather than treating the processes as ways of organizing information. The term "representation" is more suited to the stored mental model that forms the end product of these information-filtering processes, the model drawn upon in the retrieval stage of spatial memory. This final model we will refer to as the neurocognitive map. This term is chosen for two reasons. First, the model we present combines a cognitive framework for the processing stages involved in spatial representation with a corresponding set of neuroanatomical areas that underpin these cognitive activities. Second, since its use by O'Keefe and Nadel (1978), the term "cognitive map" has become loosely synonymous with the primary function of the hippocampal formation. We have therefore introduced the term "neurocognitive map" to differentiate our model's view of multiple spatial representations underpinned by an extended set of brain structures including hippocampus and several extra-hippocampal areas, and the O'Keefe and Nadel conception of "the Hippocampus as a Cognitive Map.”

\section{Spatial Strategies}

The relationship between living organisms and their environment is crucial for assuring that organism's survival (Bloch and Morange, 1997). The ability to move from one place to another allows both animals and humans means of satisfying their needs (Garling and Golledge, 2000). To understand how transitions between places are executed, one must investigate how knowledge of the environment, represented in memory, enables organisms to form and execute movement plans. Tversky (2000) suggests that, at a global level, an organism needs a mental representation of an area and that it is from this overview of space that a feasible route is determined. A route is defined as a trace of the planned or traveled sequence of path segments and turn angles that are followed in order to get from an origin to a specific destination. The process of defining a route and finding one's way along it depends on the purpose(s) of the trip, and whether it involves single or multiple stops (Golledge, 1999). Furthermore, the organism needs to know how to negotiate its route, i.e., how to maintain course while avoiding obstacles. Each of these behaviors requires different kinds of information from the world and the brain, information that is integrated by the action of spatial strategies. Although these strategies are useful in orienting in a given environment, they also contribute to the organization of gathering or encoding new incoming spatial information (Thinus-Blanc and Gaunet, 1999). Thus, constructing a spatial representation is an everyday task, and the ability to do so is important for survival. The following discussion will focus on spatial representations in adults, with a particular emphasis on environmental learning via navigation or route-based knowledge. For detailed discussions on the development of spatial representations in children, see Millar (1994) and Overman et al. (1996).

\section{Egocentric and allocentric spatial strategies}

O'Keefe and Nadel (1978) suggest there is a dichotomy between egocentric (i.e., ego-centered; body-centered) and allocentric (also referred to as exocentric, exo-centered, or environment-centered) spatial strategies. An egocentric strategy refers to the discrimination of a spatial locus with reference to the body midline, vertical visual meridian, or relative self-movement. The term has been used in at least two ways. Firstly, to refer to the encoding of spatial positions of distal objects by reference to an ego-centered coordinate system, implying a role in the cognition of large-scale spaces and secondly, in terms of the relationship of the observer to near objects, particularly objects that are within reaching distance. Egocentric space is important because it includes the region of central space to which sensory processes are most sensitively directed and the use of egocentric coordinates enables the identification of spatial loci in relation to the body midline (Foreman and Gillet, 1997). However, the body midline and vertical visual meridian are always shifting, as the organism is never completely still and the ego-centered system does not produce a stationary egocentered array, except for the briefest periods of time between successive saccadic eye movements or bodily adjustments. Thus, spatial processing becomes more complex once the organism begins to move around in space and involves memory for inter-relationships between objects in a more global or allocentric spatial framework. Consequently, compared with egocentric space, allocentric space is likely to involve a much greater memory component, and use a wider range of both perceived and nonvisible cues. Travel requires humans to activate both egocentric and allocentric processes to facilitate spatial knowledge acquisition: person-to-object relations that dynamically alter as movement takes place (egocentric referencing) and a more stable object-to-object allocentric strategy that anchors their cognitive map (Sholl, 1996).

\section{Route- and survey-based information}

There are many ways that one can learn the layout of an environment (Tellevik, 1992). When the environment is novel, altered or has not previously been experienced, possible learning strategies include active search and exploration, and a priori familiarization through the use of secondary information sources such as maps, photographs and videos etc. Two commonly described ways of learning about an environment are by acquiring route- or survey-based knowledge. Route-based knowledge is characterized as knowledge of a spatial layout from the perspective of a ground-level observer navigating the environment. We define this knowledge as any information pertaining to the environment that is acquired as a result of physical navigation through the environment. This type of information is obtained through the use of the egocentric spatial strategy discussed above, as all information is acquired with respect to the location of the viewer. Route-based environmental learning is perhaps the most common technique used by humans (MacEachren, 1992). However, an important distinction must be made between egocentric strategy and route-based information. While 
the egocentric strategy makes use primarily of visual and auditory information derived from route-based learning, this routebased knowledge contains more information than visual and auditory input; also included is knowledge pertaining to distances, angles, vectors and heading information, as well as topological information such as slopes and inclines, and vestibularbased balance and proprioceptive information. These other types of information are also accumulated through route-based learning, but are not used in the egocentric strategy. Rather, this information is drawn upon by another process, path integration, which will be discussed below.

Route-based knowledge also is differentiated from survey knowledge, which is characterized by an external perspective, such as an aerial or map-like view, allowing direct access to the global spatial layout. Evidence for a fundamental distinction between these two types of spatial information has come from the behavioral analysis of human spatial cognition (Peruch et al., 2000; Golledge, 1999; Thorndyke and Hayes-Roth, 1982; Siegel and White, 1975; Tversky, 1991). Survey-based information closely resembles the allocentric spatial strategy discussed above in that the contents of the environment are assimilated with respect to each other, irrespective of the location of the viewer. An allocentric model (like a map) of the environment appears to be built up as a derivative of physical travel through the environment. As the animal's route-based knowledge increases, an allocentric map composed of surveybased information is inferred. While this process is the normal "organic" process by which an allocentric model develops, the presence of an external or secondary source (such as an actual map, aerial photograph or other resource) can lead to the instant formation of an allocentric representation (albeit relatively impoverished) without the need for travel in the environment.

\section{Path integration}

The term path integration (PI) refers to the updating of position on the basis of velocity, temporal and acceleration information (Gibson, 1950; Loomis et al., 1999; Etienne, 1992; Mittelstaedt and Mittelstaedt, 2001). It is likely to involve an azimuthal reference (e.g., the dawn or setting sun, or a mountain range), vestibular inputs, and proprioception, possibly aided by optic flow and local features of the environment (Tversky, 2000). It involves recognizing an origin and (usually) a destination and identifying route segments, turn angles, and the sequence of segments and angles that make up the desired path. Although PI is based on route information, the internal representation underlying it is very different from what is referred to as route knowledge (Loomis et al., 1999). The internal representation of a route that has been traversed contains information about the various path segments and turns as well as off-route landmarks that might be used in subsequent piloting. In contrast, the representation underlying PI is a constantly updated abstraction derived from computations on the route information. We therefore suggest PI is a parallel process to the egocentric strategy, both of which are initiated by travel through the environment. A more detailed account of models of PI can be found in Loomis et al. (1999).

Although there is wide support for the idea that the HF is involved in PI, these studies have largely been conducted with animals (e.g., McNaughton et al., 1996; Markus et al., 1994); there is a paucity of studies examining the process of path integration in humans. However, Worsley et al. (2001) have focused on the role of the left and right temporal lobes (including the hippocampal region) in human path integration in a lobectomy study; they concluded that PI involved a number of key processes, such as establishing an initial reference point, monitoring relevant self-motion inputs, processing self-motion inputs to derive information about distance and direction traveled, and integrating of distance and directional information to derive a homing vector. Finally, spatial updating is served by a mnemonic component that tracks the outbound route and/or the distance and direction of the initial reference point, relative to the individual's current position (Worsley et al., 2001). We suggest that this latter spatio-mnemonic component is subserved by the HF, while the other processes are driven primarily by sensory systems (for a more detailed discussion, see the section, Model of Spatial Representation Acquisition).

\section{Neural Substrates of Route- and Survey-Based Learning}

Mellet et al. (2000) found that survey/telegraphic imagery elicited activation in the right $\mathrm{HF}$ alone, but found bilateral activation of parahippocampal gyrus (PHG) for route imagery. However, this study focused on previously learned environments and not the encoding of novel environments. More recently, Shelton and Gabrieli (2002) investigated the encoding of two different VR environments (one from a route and the other from a survey perspective) in a group of healthy volunteers. They investigated if two types of spatial information were distinguished in the brain by recruitment of different areas or rather by differential activation within the same cerebral spatial processing network. Route and survey encoding led to different mental representations of the spatial environments, as depicted in the ways in which maps of the environments were drawn. However, both types of encoding yielded almost equivalent accuracy in tasks measuring map-drawing and recognition. This important finding implies that the observed differences in brain activation could not be attributed to encoding success or failure, but instead reflected differences in the underlying processing. Route encoding activated bilateral MTL (including parahippocampal cortex and posterior HF), postcentral gyrus, right posterior cingulate and left medial frontal gyri (MFG), bilateral fusiform and inferior temporal gyri (ITG) and the posterior superior parietal cortex (see Fig. 2). Survey encoding resulted in greater activation than route in a subset of these areas, namely bilateral fusiform and inferior temporal gyri and posterior superior parietal cortex. The authors proposed that the increased activation of a specialized subset of regions by survey encoding implies that the survey-based system evolved as a secondary system within a larger route-learning system. This idea 
A

\section{Route > Survey}
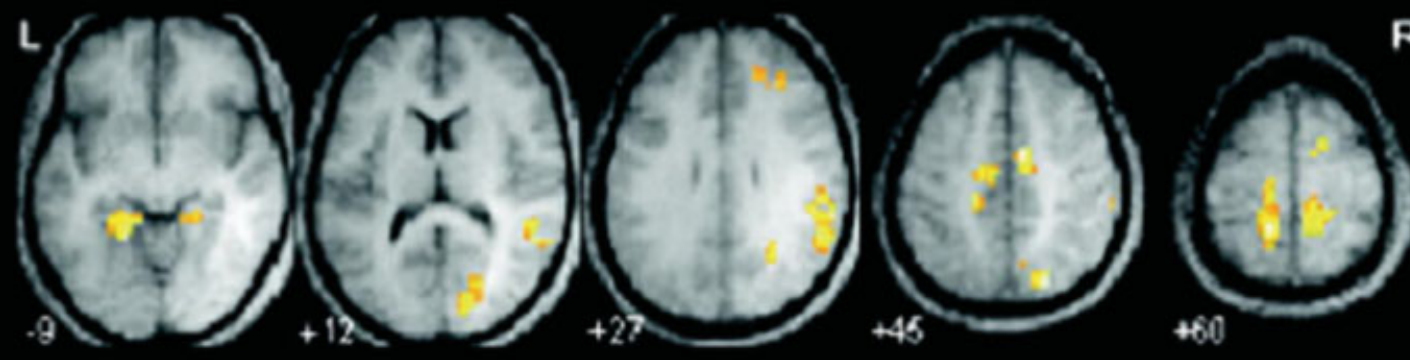

\section{Survey > Route}

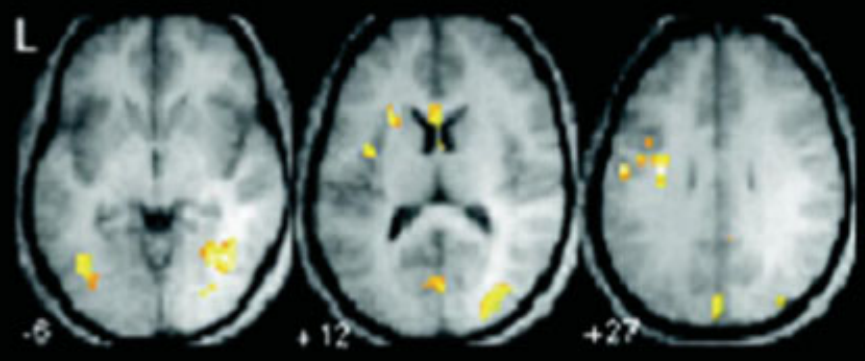

445
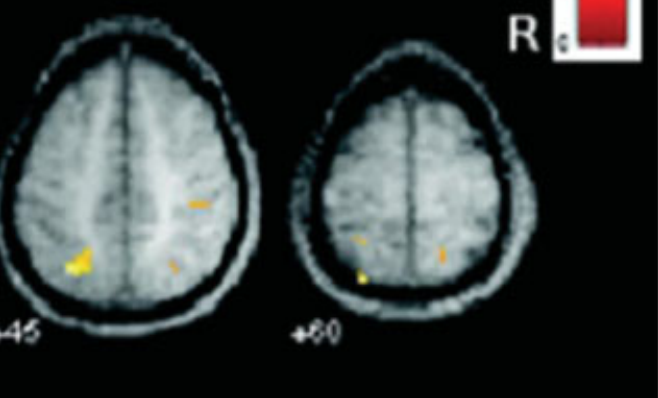

B

\section{Pest-central gyerus (BA.5.7)}

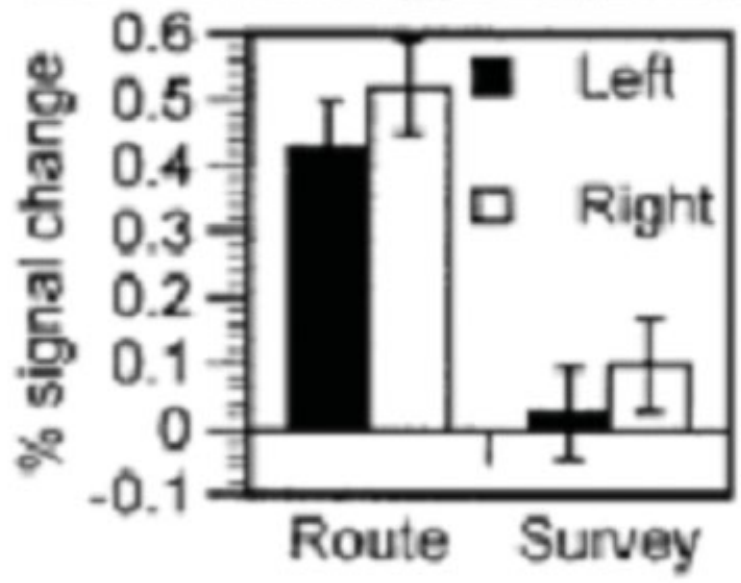

FIGURE 2. A: Areas of significant activation for direct comparisons of route and survey encoding. B: Mean percentage of signal change as a function of encoding type for clusters that had greater activation for route encoding than for survey encoding. Error bars reflect \pm 1 SEM. (Reproduced from The Journal of Neuroscience 22:2711-2717,

supported earlier developmental research (e.g., Siegel and White, 1975), showing that children develop route learning before survey learning. Shelton and Gabrieli also stressed an

\section{Medial temporal lobe}

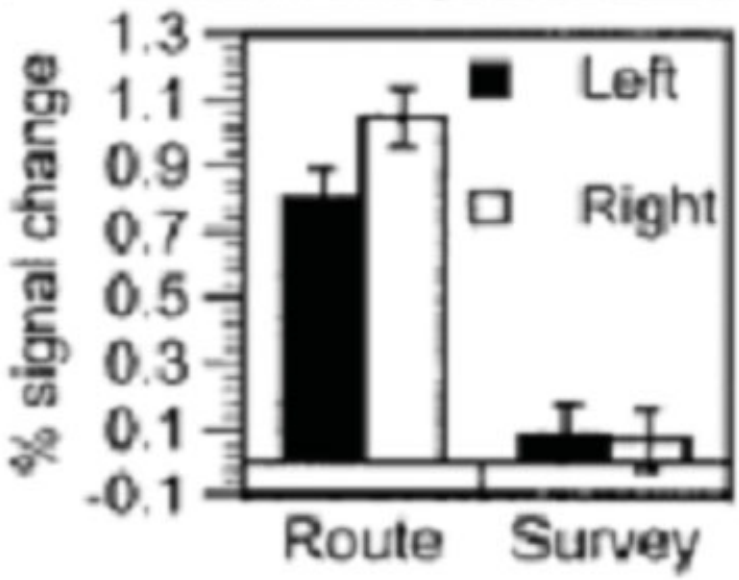

Shelton and Gabrieli, 2002, Neural correlates of encoding space from route and survey perspectives, with permission from the Society for Neuroscience.) [Color figure can be viewed in the online issue, which is available at www.interscience.wiley.com.]

important difference between route and survey encoding that may be critical to the observed brain differences: route perspectives facilitate a sense of immersion in the environment, while 
no such experience was reported for survey encoding, where participants describe the condition as a map.

\section{Neural Substrates of Path Integration: Vestibular System}

Many studies have focused on the vestibular system and its role in human navigation: there are well-documented vestibular-hippocampal interactions in rats and monkeys (e.g., O'Mara et al., 1994; Smith, 1997; Taube, 1995; Sharp et al., 1995; Gavrilov et al., 1995; Wiener et al., 1995). In an fMRI study by Lobel et al. (1996) caloric stimulation was applied to the vestibular apparatus of seven subjects by injection of cold water into the left ear canal and this was compared with a rest condition. The results showed bilateral activation of the HF, with this activation being more consistently reported for the right side.

The contribution of the vestibular system to the orientation and localization of the body in space after passive displacement in animals and humans has long been suggested (Beritoff, 1965; Potegal, 1982; Telford et al., 1995). Beritoff (1965) observed that blindfolded deaf-mute children with lesioned labyrinths could not retrace the route along which they had been taken, when compared with normal children. His conclusion that the vestibular organs were necessary for navigation in the dark has been supported by more recent studies (e.g., Ito et al., 1992; Ito et al., 1995; Glauser et al., 1994). Takei et al. (1997) also found that patients with unilateral vestibular damage tended to deviate or even fall to the inside of a circular path when they were turned toward the affected side. In contrast, normal adults could remember a previously walked circular path and reproduce the trajectory with reasonable accuracy when visual and auditory cues were eliminated (Takei et al., 1996). This pattern of results may be explained by the existence of an independent functional representation which contains vestibular/angular trajectory information and operates independently of visual and auditory information.

Berthoz and colleagues have also shown that humans have the capacity to compute actual displacement during passive whole-body motion on the basis of vestibular signals (Berthoz et al., 1995; Berthoz, 1997; Berthoz, 1999). These investigators proposed that internally-simulated action is a fundamental element of perception and spatial memory and that somatosenory and vestibular information is important for keeping track of turning motion. These sensory cues are used to infer headings and spatial relations of surrounding objects (Berthoz et al., 1995; Takei et al., 1996; Takei et al., 1997). Berthoz (1999) suggested that vestibular patients have a specific deficit of the head-direction cell system (Taube, 1998), which renders them unable to store directional information. Furthermore, in the absence of vision or in the presence of conflicting visual cues, this results in a deficit in the evaluation of direction. However, these ideas have been challenged; Burgess et al. (2002) have suggested that self-motion produces idiothetic signals that automatically update the internal spatial representation of an environment in a more accurate way than one's ability to perform the equivalent mental rotation deliberately. However, in contrast with Berthoz, they propose that this process involves updating a viewpoint within a cognitive mental model that is independent of vestibular or proprioceptive signals. The model presented in the section, Model of Spatial Representation Acquisition, resolves this conflict by considering path integration and egocentric strategy as independent parallel processes: therefore, Berthoz's point addresses the functional spatial construct derived from path integration, while Burgess et al. refer to the egocentric model produced via egocentric strategy.

\section{COGNITIVE MAPPING IN HUMANS}

\section{How Humans Record and Represent Environments}

The result of the convergence of route-based and surveybased information gathered via egocentric and allocentric strategies (and path integration) is a composite mental representation of the environment containing both types of knowledge. According to Garling and Golledge (2000), the end-product of the selection processes described above is a memory representation or cognitive map of a particular environment. The term "cognitive map" was first coined by Tolman (1948) and since then, the term has come into wide usage within this specialized literature and is usually taken to refer to the internal representations of perceived environmental features or objects and the spatial relations among them (Golledge, 1999). Cognitive maps are considered the most frequently used representational form of experienced environments, in addition to the commonly stored knowledge of individuals or societies, and there is emerging evidence that maps are cultural universals (Uttal, 1994). A cognitive map is more abstract than route knowledge and has image-like properties that allow an individual to plan effective short-cuts and detours and to quickly estimate distances and bearings from any location within the map to any other such location (Peruch et al., 2000). Cognitive maps consist of points, lines, areas surfaces and direction which are learned, experienced and recorded in quantitative and qualitative forms (Garling and Golledge, 2000).

\section{Landmarks in cognitive mapping}

From the point of view of wayfinding, the most important features of views are physical landmarks. They may be defined in a number of ways, such as the strategic foci toward or away from which one travels, intermediate foci on courses and routes that assist spatial decision making, or significant physical structures, buildings or culturally defined objects that stand out from their surroundings (Golledge, 1999). Landmarks are often noticed and remembered because of dominance of visible form, peculiarity of shape or structure or because of sociocultural significance. The traditionally used concept of a landmark has two distinct components: it is capable of attracting attention, being commonly recognized by many people and in some cases, landmarks are accrued significance in an idiosyncratic 
way (e.g., place of work). Landmarks usually act as anchor points for organizing other spatial information into a layout and may thus act as primary organizing features in cognitive maps (Couclelis et al., 1987). Along routes when choices have to be made at intersections, specific memorization of a landmark can help specify when to turn and which route to select next. Such goal-based decision making processes would be subserved by the prefrontal cortex, while the processing of the landmarks themselves would involve the areas associated with the egocentric strategy, outlined above.

Daniel and Denis (1998) found that in asking students to describe three routes on a university campus, about $17 \%$ of utterances prescribed an action, 36\% prescribed an action with respect to a landmark, 33\% introduced a landmark and 12\% described a landmark. Overall, landmarks seem to be selected on the basis of visibility, pertinence, distinctiveness, and permanence. They served several functions: to signal the place of action change, to locate other landmarks and to confirm the route (see also Heft, 1996, who showed that routes could be learned by viewing film segments rather than by language).

Neuropsychological evidence for the existence of a specialized area within extrastriate cortex which is sensitive to "landmark" stimuli has come from a subset of patients who suffer from topographical disorientation, a disorder characterized by a set of neuropsychological deficits following different, isolated brain lesions (Aguirre and D'Esposito, 1999). One such deficit is landmark agnosia (Whiteley and Warrington, 1978; Levine et al., 1985; Aguirre et al., 1998) and is separate from other agnosias such as general object agnosia, or prosopagnosia. Aguirre et al. (1998) suggested that landmark agnosia patients had sustained damage to a cortical region that was specialized for the perception of visual stimuli with a particular orienting value (i.e., isolated landmarks, as well as "scenes" of the environment). From their fMRI study with healthy controls, Aguirre et al. found a region in the anterior area of the right lingual gyrus, which they tentatively dubbed "lingual landmark area," that appeared to be maximally sensitive to buildings as opposed to objects or faces. Importantly, this critical area is adjacent to the parahippocampal gyrus; the anatomic boundary between the superior lingual gyrus and the parahippocampal gyrus is poorly defined (Duvernoy, 1991). This led Aguirre and colleagues to propose that the region identified in their studies happened to be the same as that found by Epstein and Kanwisher (1998), which they termed the "parahippocampal place area," which is presumably derived from the hippocampal "view cells" found in the primate HF (Rolls and O'Mara, 1995). From these studies, the evidence for the role of the PHG in an egocentric/landmark-based representation is quite compelling.

\section{Limitations of Cognitive Maps}

Most conceptions of cognitive maps recognize that not all the information in the environment need be represented; much of it is left out and what is included is schematized, simplified or idealized (Tversky, 2000). At later stages of acquisition, when the cognitive map guides choices of places, paths, and wayfinding decisions, further limitations are applied to the sub- sequent selection of information about the environment. Therefore, the cognitive map is unlikely to be fully complete and many inaccuracies are never corrected (Garling and Golledge, 2000). Incompleteness of cognitive maps resulting from deliberate selection of available information leads to a hierarchical organization of information in memory, and the use of humanbased methods in creating spatial representations implies that humans' cognitive maps can be error prone, leading to distortions and fragmentation (Golledge, 1999).

\section{HIPPOCAMPAL FORMATION AND SPATIAL REPRESENTATION}

A large literature links the HF to certain aspects of human and animal spatial representation and navigation. These studies avail of a number of useful experimental paradigms in combination with lesion or imaging techniques. The most commonly used paradigms include virtual reality (VR) tasks, real-world navigation, viewpoint-dependent studies, passive navigation, and blind navigation. The findings from each approach are reviewed in turn in this section.

\section{Virtual Reality Tasks}

VR elicits a strong sense of "presence" compared with tabletop tests (Held and Durlach, 1992): the subjective experience of being in one place when one is physically in another. Witmer and Singer (1994) found significant correlations between "presence" and performance in VR environments; in consequence, Burgess et al. (2002) have suggested that there is a good correspondence between the spatial knowledge acquired in a real world and a model of that environment in VR. However, in order to be effective, VR environments must contain features of real-world environments such as realistic landmarks. The majority of VR paradigms involve the participant navigating through a city or town (e.g., Gaunet et al., 2001; Spiers et al., 2001a,b), although some researchers have used virtual maze paradigms, adapted from tasks such as the Morris water maze (usually associated with studies of rodent spatial representation), to investigate human navigation (e.g., Aguirre et al., 1996; Maguire et al., 1998; Astur et al., 1998; Sandstrom et al., 1998).

\section{VR town navigation}

From their studies focusing on navigation in VR towns, Maguire et al. (1998) hypothesized that the output of the right $\mathrm{HF}$ was a vector that continuously pointed to the goal direction (consistent with Burgess and O'Keefe's (1996) model of the rat $\mathrm{HF}$ ). Left hippocampal activation was associated with successful navigation, but was not as sensitive to accuracy in navigation as the right $\mathrm{HF}$. Maguire and coworkers suggested that the left HF possibly had a less spatial and more verbal role in memory. This assumption is consistent with a number of studies (e.g., Astur et al., 2002, Fig. 3; Worsley et al., 2001, Fig. 4) that have found either the left or right MTL lesions in 
A

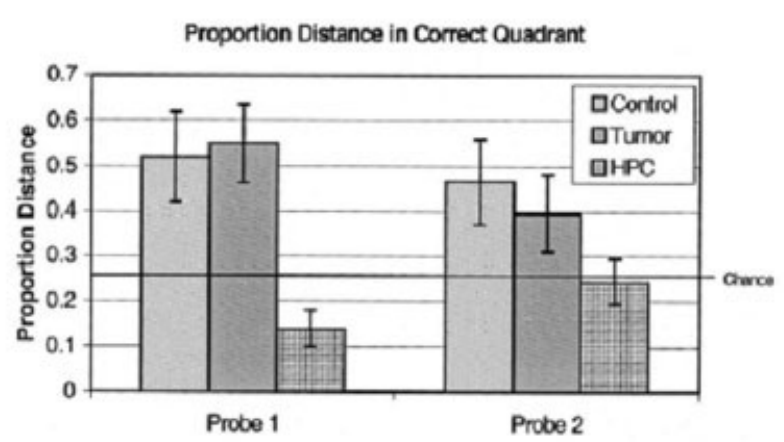

B

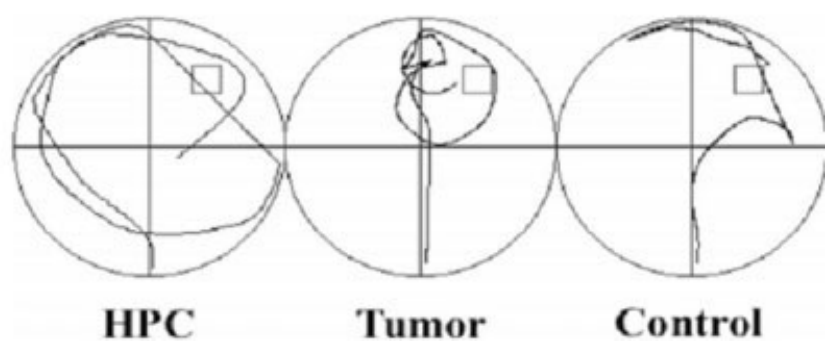

FIGURE 3. A: Proportion distance traveled in the quadrant of the pool where the hidden platform was located for the three groups. For the first probe, the HPC group shows significantly less of a preference than the other two groups. For the second probe, the HPC group again shows significantly less of a preference than the Control group, but the tumor group does not differ from the other two groups. B: Probe trial swim paths from the first probe trial of the median individual in each group, as determined by probe trial performance. Note the lack of a preference for the correct quadrant by the individual from the HPC group. (Reproduced from Behavioural Brain Research 132:77-84, Astur et al., 2002, Humans with hippocampus damage display severe spatial memory impairments in a virtual Morris water task, with permission from Elsevier.)

humans negatively affect navigation (although right-sided impairments were more generally associated with spatial memory deficits). This issue will be revisited in the presentation of our model below.

\section{VR maze navigation}

A principal finding from these studies has been that successful navigation in a virtual maze is associated with increased activity in the parahippocampal gyrus but not the HF, implying that the parahippocampal gyrus is a key neural structure in human spatial mapping (Aguirre et al., 1998). Additionally, Epstein and Kanwisher (1998) found parahippocampal cortex activation in their investigation of memory for scenes, when presented as route-perspective images (see also Nakamura et al., 2000). The parahippocampal gyrus may support the processing of spatial information that is present in the visual scene, for example, extracting the distances to the nearest landmarks or boundaries (Burgess et al., 2002; Aguirre et al., 1998). A recent study by Astur et al. (2002) used a VR Morris water maze task and reported severe spatial memory impairments in a group of right and a group of left hippocampal patients compared with age-matched patients with resections outside of the hippocampal region and age-matched healthy controls. Astur et al. suggest that these deficits may be attributed to the hippocampal patients' inability to integrate visual motion (optokinetic) and vestibular information in order to engage successfully in path integration or cognitive mapping strategies.

\section{Real-World Navigation}

In general, imaging studies have repeatedly shown activation of the HF proper in the recall and generation of routes through a real city and recalling a prelearned route in the real world (Burgess et al., 2002). Increases in hippocampal activity have been consistently reported when subjects learned how to navigate through a real town (Maguire et al., 1996) (Fig. 5A), by recalling routes through a real city (Maguire et al., 1997) or by recalling a route learned in the real world before scanning took place (Ghaem et al., 1997). Consistent with the observations from VR town studies, activation of the right $\mathrm{HF}$ in real-world navigation tasks is associated with accurate wayfinding, whereas the precise functions served by the left hippocampal activation during spatial behavior remain unclear (Maguire et al., 1998) (Fig. 5B). In a PET study using a Mental Simulation of Routes (MSR) task, Ghaem et al. (1997) showed that engaging in mental navigation along a learned, real-world route activated the right middle hippocam-

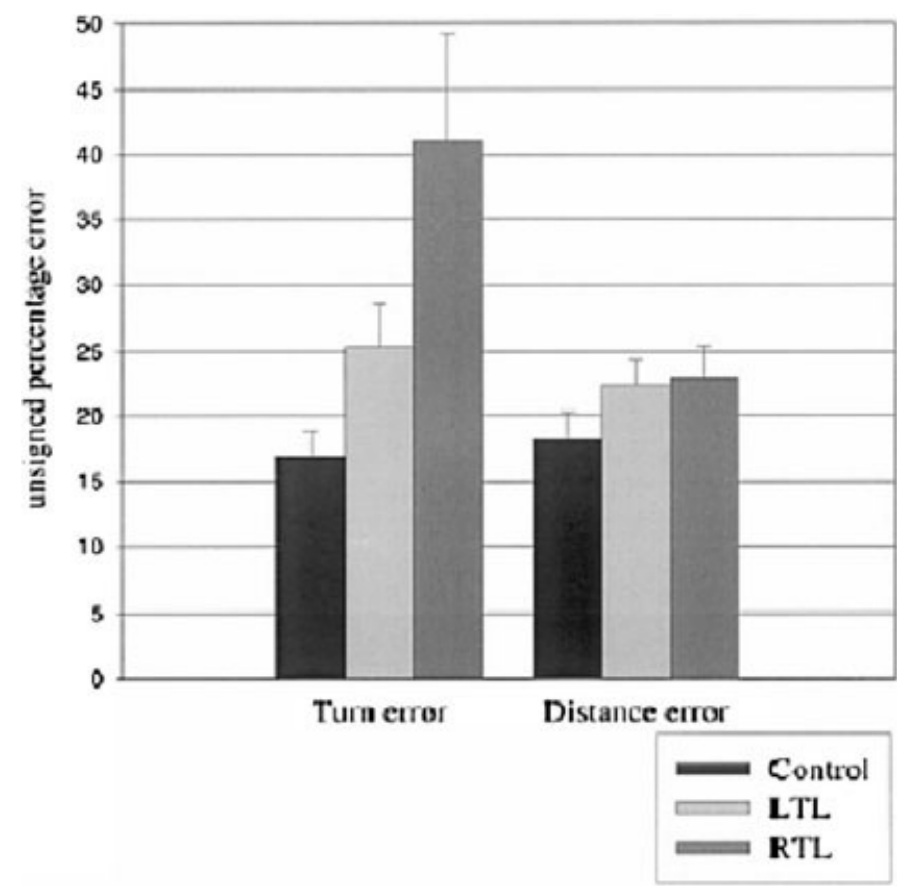

FIGURE 4. Homing vector test: turn error (unsigned percentage) and distance (unsigned percentage) error in the control, LTL, and RTL groups. (Reproduced from Neuropsychologia 39:452464, Worsley et al., 2001, Path integration following temporal lobectomy in humans, with permission from Elsevier.) 
A

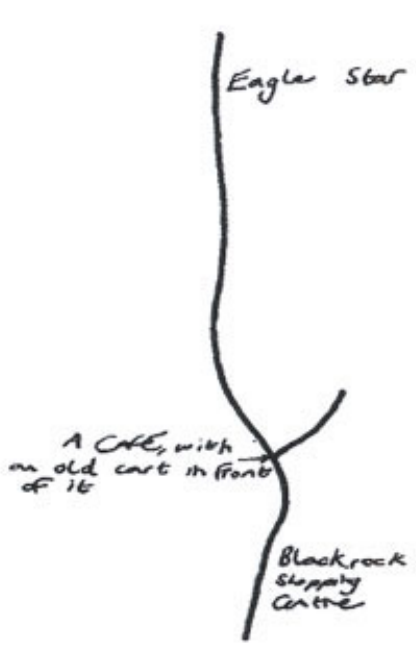

right temporat-lobe surgery subject

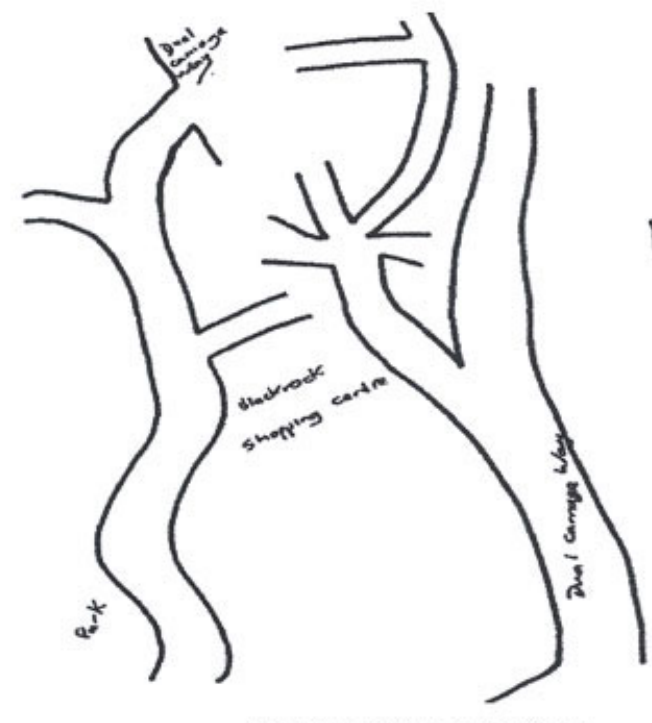

left temporal-lobe surgery subject

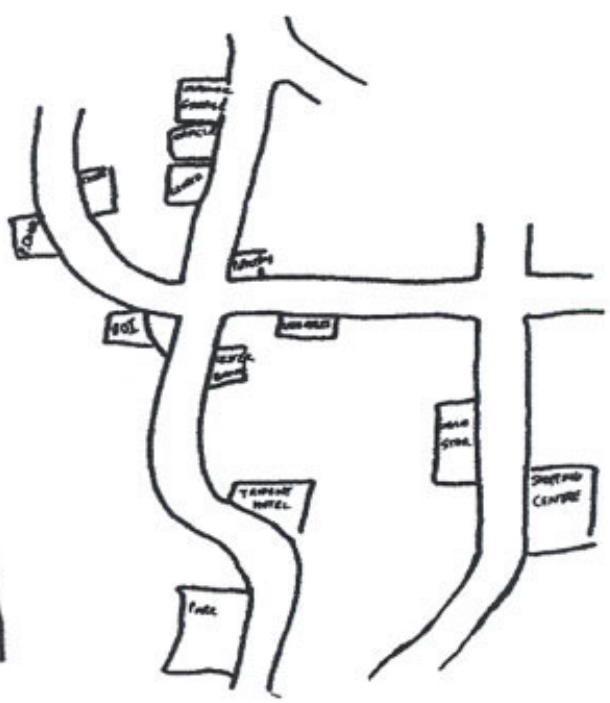

normal control subject

B
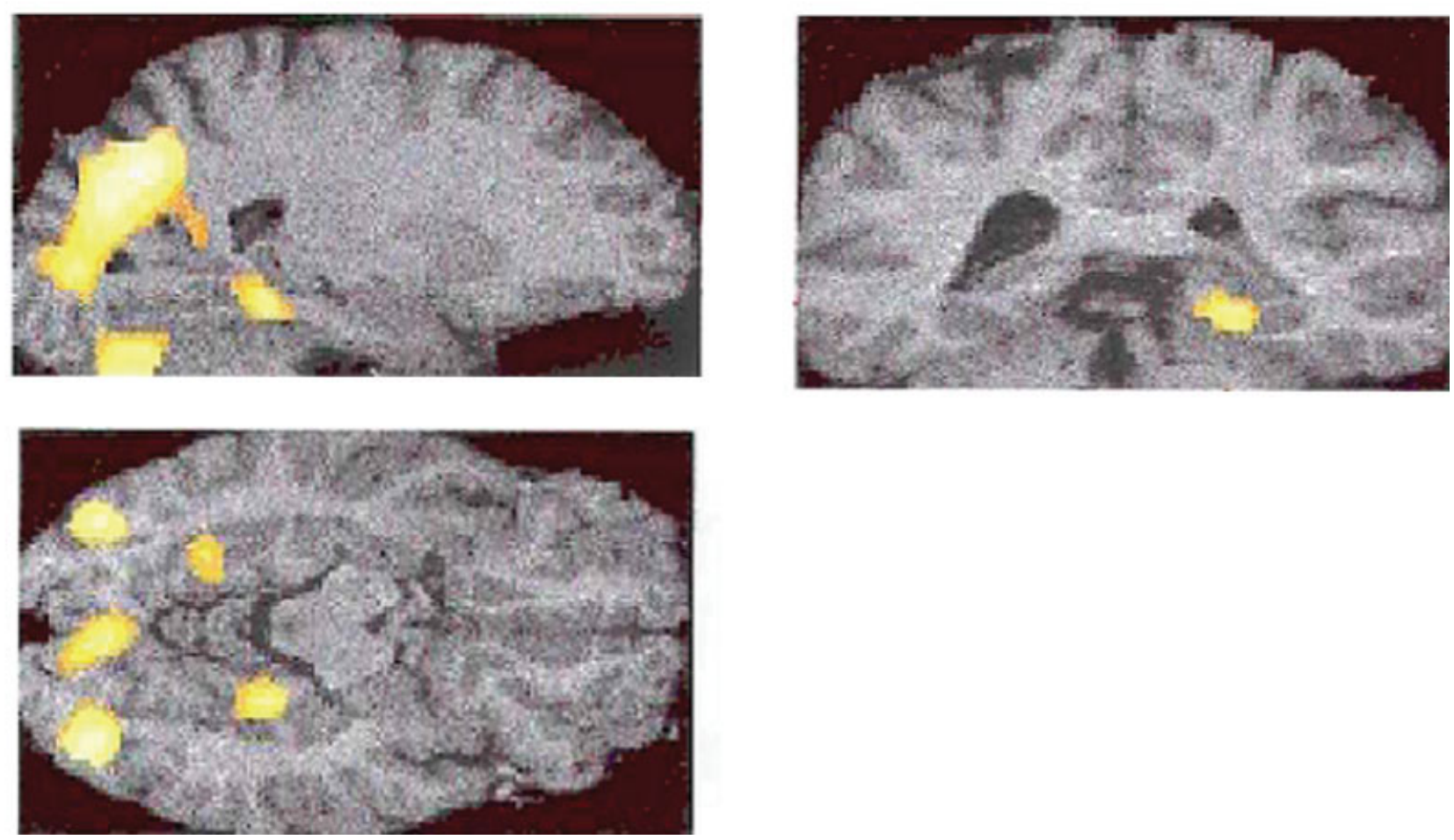

FIGURE 5. A: Examples of sketch maps of the overlapping routes produced by subjects. (Reproduced from Neuropsychologia 34:132-136, Maguire et al., 1996, Topographical disorientation following unilateral temporal lobe lesions in humans, with permission from Elsevier.) B: Regions of significantly greater activation during the exploration of the environment with objects compared with random color/texture images. Superimposed onto a template MRI scan at the level of the peak activation. (Reproduced from Journal of Cognitive Neuroscience 10:61-76, Maguire et al., 1998, with permission from the Massachusetts Institute of Technology.) [Color figure can be viewed in the online issue, which is available at www.interscience.wiley.com.] 
pal areas, as well as the posterior hippocampal areas bilaterally. Visual recall elicited bilateral activation of the middle hippocampal regions, the left inferior temporal gyrus, and left posterior hippocampal regions. Ghaem et al. (1997) suggested that the left HF was involved in the recall of the relation between whole-body movements and the environment. The involvement of left and right $\mathrm{HF}$ in this task again supports the model presented below and its proposed loci for the functional representation.

\section{Viewpoint-Dependent Studies}

VR paradigms have also been designed to test the effects of manipulating the subjects' viewpoint on their subsequent performance of different tests of spatial representation. King et al. (2002) suggest such studies have yielded three important findings. First, automatic processes may support the accommodation of a change of viewpoint within one's mental representation of object location (Wang and Simons, 1999). Second, Christou and Bulthoff (1999) showed that the important factor in these studies was the movement of the viewpoint relative to the participants' cognitive model of the world rather than actual movement and the attendant vestibular and proprioceptive feedback or viewed movement of the objects relative to the subject. Finally, when subjects were shown a scene of scattered objects from one viewpoint and given a recognition memory test using scenes from a different viewpoint, their response latencies varied linearly with the angular difference between the views (Diwadkar and McNamara, 1997; see also Easton and Sholl, 1995). In sum, the use of an allocentric process capable of arbitrary manipulations of viewpoint within 3D space appears to be specifically dependent on the HF (Vargha-Khadem et al., 1997).

\section{Passive Navigation}

VR paradigms have also been used to study passive navigation. Gaunet et al. (2001) examined spatial memory of a virtual city using active, passive and snapshot exploration methods. They found that accuracy in scene recognition and estimation of the direction of the origin of the path was not influenced by the exploration conditions. In contrast, however, the reproduction of the path shape was affected, with greater total distance and angle reproduction error scores following snapshot exploration than active and passive explorations. This finding highlights the importance of continuous updating in learning a novel environment and implies that a continuous stimulation of visual exploration enhances the ability to reproduce the shape of a path previously traveled. Being able to engage in continuous updating requires an ability to fill in the gaps between snapshots based on the interpolation of one's position related to the previous snapshot. Gaunet et al. (2001) proposed that during snapshot exploration, the processes used by participants (either creating a mental frame of reference or engaging displacement updating) were not adequate for path shape reproduction.

\section{Blind Navigation}

Most research on human cognitive mapping has focused on vision since it conveys the important information about the spatial layout of the near and distant environment, and also the details contained in maps, and aerial photographs and so forth. However, it is clear that some blind persons are able to navigate and to form cognitive maps of the environment using the remaining information available to them (audition, olfaction, touch, proprioception and efference copy; Peruch et al., 2002). A number of researchers (e.g., Loomis et al., 1993; Rieser, 1989; Berthoz et al., 1995) have focused on the ability of sighted individuals to navigate without the use of vision (see also Gaunet and Thinus-Blanc, 1996; Thinus-Blanc and Gaunet, 1997; Ungar, 2000). Thomson (1983) showed that humans with eyes closed can reach a previously seen target on the floor several meters away. Rieser (1989) and Loomis et al. (1993) have investigated the ability of persons, with and without vision, to imagine standing at a known location and to indicate by pointing or walking toward, the direction of obscured locations. Accuracy was seen to vary considerably depending on the participant's familiarity with the test area. This implies that in unfamiliar environments, if directions are not well known, large errors in wayfinding may occur either in terms either of actual travel or in verbally describing or sketching where to travel. Thus, information about step length, derived from proprioceptive or outflow motor command signals, as well as from vestibular signals can contribute to the updating of the mental representation of the subject's location in space and allow for path integration (Mittelstaedt and Glasauer, 1991; Glasauer et al., 1994).

\section{Paradigms: Overview}

VR and viewpoint-dependent tasks allow for the presentation of egocentric route-based information to be presented in the absence of path integration or vestibular input, thereby allowing the egocentric and allocentric representations of the cognitive map to be derived while the functional model will be impoverished. Real-world navigation will allow all modelsegocentric, allocentric, and functional-to be developed as occurs naturally. Passive navigation paradigms allow for vestibular information to be assimilated without proprioceptive feedback from muscles and joints, which in turn will allow the different facets and anatomical substrates of the functional representation to be disentangled. Finally, blind navigation studies permit us to investigate the process of path integration and the functional representation when the primary input to the egocentric strategy-vision-is deprived.

\section{MODEL OF SPATIAL REPRESENTATION ACQUISITION}

A substantial theoretical and empirical consensus suggests that the $\mathrm{HF}$ is involved in human spatial representation, navi- 


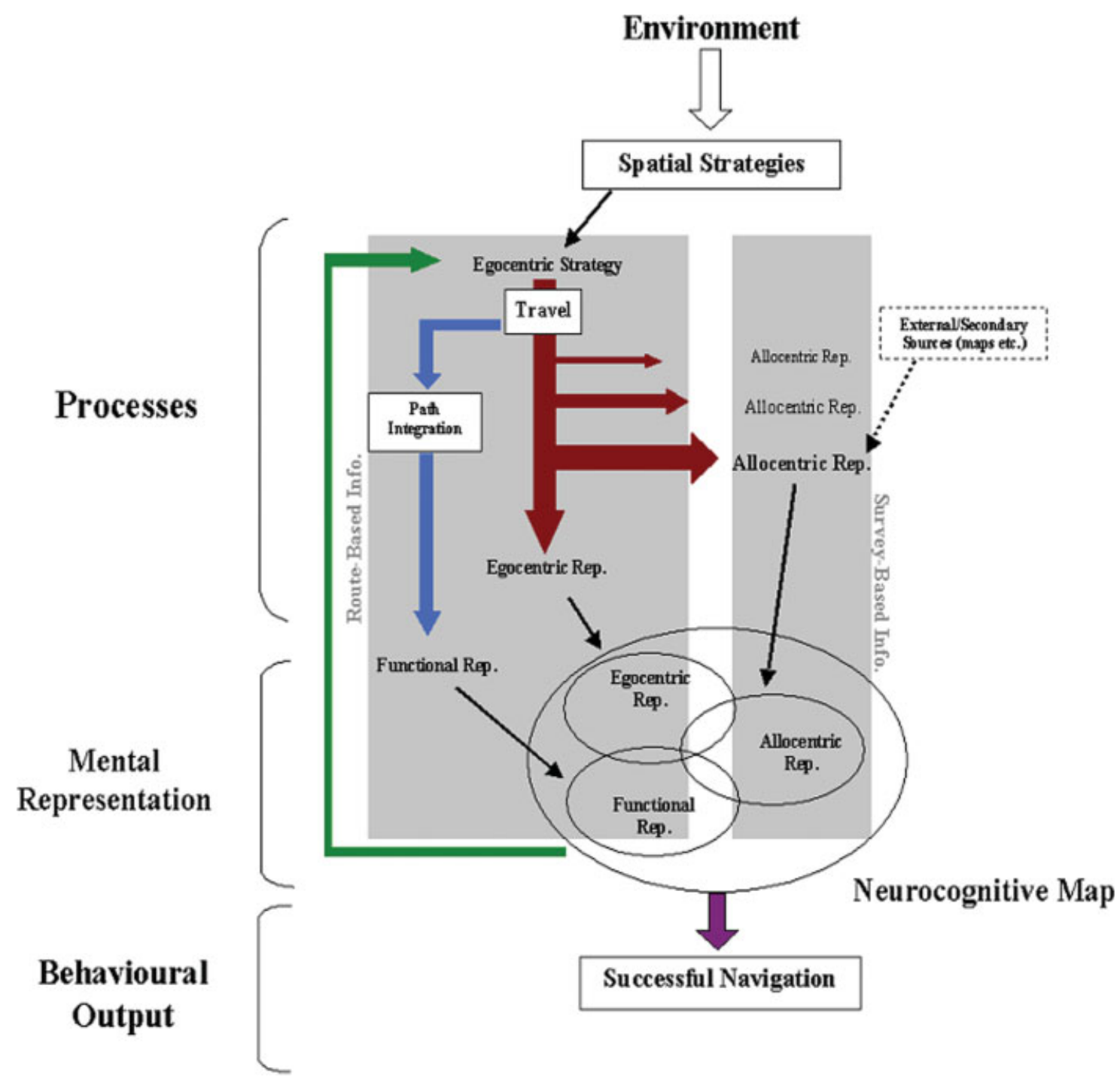

FIGURE 6. Flow diagram representing a new model that outlines how the different processes interact and segregate the different forms of information in producing the unified cognitive map. [Color figure can be viewed in the online issue, which is available at www.interscience.wiley.com.]

gation, and thus the acquisition of cognitive maps. We suggest that spatial representation in humans involves several complementary processes whereby information from the external environment is collected, segregated, and combined to form mental models of the world (which we term spatial constructs) in the form of separate and parallel person-centered and object-centered representations, as well as a representation containing path integration information. We present also a unified model of the operation of this system: information from the environment is organized by spatial strategies (Fig. 6); in the absence of an external secondary source of allocentric information (e.g., an ordnance survey map), egocentric strategies are employed to process route-based input. As soon as the organism begins to travel through the environment, the (mainly visual) egocentric strategy builds up an egocentric model/spatial construct that becomes increasingly sophisticated as more locations are visited. At the same time, the parallel process of path integration assim- ilates distance, angle, vector, vestibular, and other route-based information to develop what we term a functional representation of space. This spatial construct contains information on direction, angle, distance and terrain that will be drawn upon when the organism needs to plan and execute a route, with a less emphasis on visual aspects of active travel such as optic flow. Meanwhile, the successive gathering of route-based information leads to a sophisticated allocentric spatial construct being incrementally generated containing survey-based knowledge. Our central theoretical claim is that these three (egocentric, functional, and allocentric) spatial constructs are combined in the mental representation of the environment, which we term the neurocognitive map to emphasize its extension through a series of brain structures culminating in HF and thence onward to mnestic brain structures. Any further travel through the environment will lead to the updating of these representations via a feedback loop that will reactivate the egocentric strategies and the process will 
TABLE 1.

Summary of Predictions Based on This Model, Organized Thematically and Presented So That They Are Readily Testable Experimentally

\begin{tabular}{|c|c|c|}
\hline Prediction & Structures implicated & Tasks \\
\hline \multicolumn{3}{|l|}{ Behavioral } \\
\hline $\begin{array}{l}\text { Reproduction of navigated environment impaired when } \\
\text { participants navigate blindfolded. }\end{array}$ & & $\begin{array}{l}\text { Map drawing } \\
\text { Blind navigation }\end{array}$ \\
\hline $\begin{array}{l}\text { Blindfolded navigation disrupted when participants are } \\
\text { passively propelled through an environment; } \\
\text { reproduction of navigated environment impaired when } \\
\text { participants navigate blindfolded. }\end{array}$ & & $\begin{array}{l}\text { Passive/active travel } \\
\text { Blind navigation }\end{array}$ \\
\hline $\begin{array}{l}\text { Where the neurocognitive map derives from a schematic } \\
\text { map, we predict poorer navigation performance (based } \\
\text { on recall of that map) than in cases where the } \\
\text { representation was derived from purely egocentric } \\
\text { information or normal path integration. }\end{array}$ & & $\begin{array}{l}\text { VR navigation } \\
\text { Blind navigation }\end{array}$ \\
\hline \multicolumn{3}{|l|}{ Cognitive systems } \\
\hline \multicolumn{3}{|l|}{ Systems-Anatomical } \\
\hline $\begin{array}{l}\text { The egocentric strategy uses mainly visual information, but } \\
\text { in the absence of visual cues, auditory, tactile, or olfactory } \\
\text { information will be employed, although in these cases an } \\
\text { egocentric model of inferior resolution will result. }\end{array}$ & $\begin{array}{l}\text { Visual system } \\
\text { Auditory system } \\
\text { Somatosensory system } \\
\text { Olfactory system }\end{array}$ & Real-world/VR navigation \\
\hline $\begin{array}{l}\text { The egocentric construct will be based largely on distal } \\
\text { landmark information derived from activity in } \\
\text { fusiform, inferior temporal and posterior parietal areas. }\end{array}$ & $\begin{array}{l}\text { Fusiform gyrus } \\
\text { Inferior temporal gyrus } \\
\text { Posterior parietal }\end{array}$ & VR navigation \\
\hline $\begin{array}{l}\text { The functional representation will be reliant on a } \\
\text { subset of MTL areas specialised for the processing of } \\
\text { vestibulomotor information; it will draw primarily } \\
\text { upon vestibular and proprioceptive information, } \\
\text { with lesser inputs from visual and somatosensory } \\
\text { areas. The right hippocampal formation may be a } \\
\text { strong candidate area for the functional model. }\end{array}$ & $\begin{array}{l}\text { Right HF } \\
\text { Left HF } \\
\text { Vestibular system } \\
\text { Parietal somatosensory } \\
\text { Sensorimotor areas for } \\
\quad \text { proprioception } \\
\text { Dorsal visual areas }\end{array}$ & Blind navigation \\
\hline \multicolumn{3}{|l|}{ Process-Anatomical } \\
\hline $\begin{array}{l}\text { Encoding of functional information will activate } \\
\text { areas such as the vestibular system, parietal } \\
\text { somatosensory, and proprioceptive areas and } \\
\text { the dorsal visual stream as well as MTL. }\end{array}$ & $\begin{array}{l}\text { Vestibular system } \\
\text { Parietal somatosensory } \\
\text { Sensorimotor areas for } \\
\quad \text { proprioception } \\
\text { Visual areas } \\
\text { MTL }\end{array}$ & Real-world navigation \\
\hline $\begin{array}{l}\text { Retrieval of functional knowledge will activate bilateral HF, } \\
\text { with greater activation in the right hemisphere. }\end{array}$ & $\begin{array}{l}\text { Right HF } \\
\text { Left HF }\end{array}$ & Blind navigation \\
\hline
\end{tabular}


TABLE 1. (Continued)

Prediction
Encoding of the egocentric representation will activate a
collection of areas.
collection of areas.

Retrieval of information from the egocentric model will then engage bilateral HF and the parahippocampal gyrus/lingual gyrus.

An allocentric representation encoded due to physical travel will activate bilateral fusiform gyrus, ITG, and posterior superior parietal cortex. However, when the representation is derived from artificial means such as a map, these areas will likely not be activated, as they are a subset of the egocentric acquisition network.

Retrieval of allocentric representations (induced, for example, by drawing overhead maps of the environment) will result in activation of HF and parahippocampal gyrus.

HF is important for the acquisition of egocentric and allocentric spatial constructs, but not functional.

Anatomical connectivity

Increases in strength of connectivity (or correlations in inter-areal activity) between bilateral HF and the areas involved in the path integration system during the encoding of the functional representation of the environment.

Egocentric encoding areas, including ITG, fusiform gyrus, and middle frontal gyrus should show increased strength of connectivity (or correlations in inter-areal activity) with bilateral HF and PHG.

Allocentric acquisition areas (bilateral fusiform gyrus, ITG, and posterior parietal) will show increased strength of connectivity (or correlations in inter-areal activity) with HF and PHG. However, if the allocentric model is derived from a secondary source, no such connectivity changes will be seen.

Behavioral-Anatomical

If participants navigate via a route of their own choosing through an environment with multiple, equally effective routes to a goal destination, we predict that this will cause activation of prefrontal areas compared to a comparable task in which no alternate routes are available.

The egocentric pathway can be disrupted by removing visual input, while path integration can be hampered by vestibular disruption through caloric stimulation or spinning. In each case, the spatial construct should not be affected by disruption of the other pathway.

The allocentric spatial construct derives primarily from the egocentric strategy; thus, disrupting the cortical substrates of egocentric spatial processing will result in a less developed allocentric representation than would disruption of the path integration system.
Structures implicated

Tasks

Right and left MTL-Postcentral gyrus, posterior superior parietal cortex, fusiform gyrus

ITG

Right and left HF

PHG

Lingual gyrus

Bilateral fusiform gyrus

Inferior temporal gyrus

Superior parietal

Real-world/VR navigation

Landmark recognition

Real-world/VR navigation

$\mathrm{HF}$

PHG

Map drawing

HF

HF-PI system

Real-world navigation

ITG, FFG, MFG: HF and PHG

Real-world/VR navigation

ITG, FFG, PPC: HF and PHG

Real-world/VR navigation

PFC + egocentric and PI areas

Egocentric areas

Functional areas

Real-world navigation with caloric stimulation

Egocentric areas

Real-world navigation

Functional areas
Blind navigation

Map drawing 


\section{Environment}

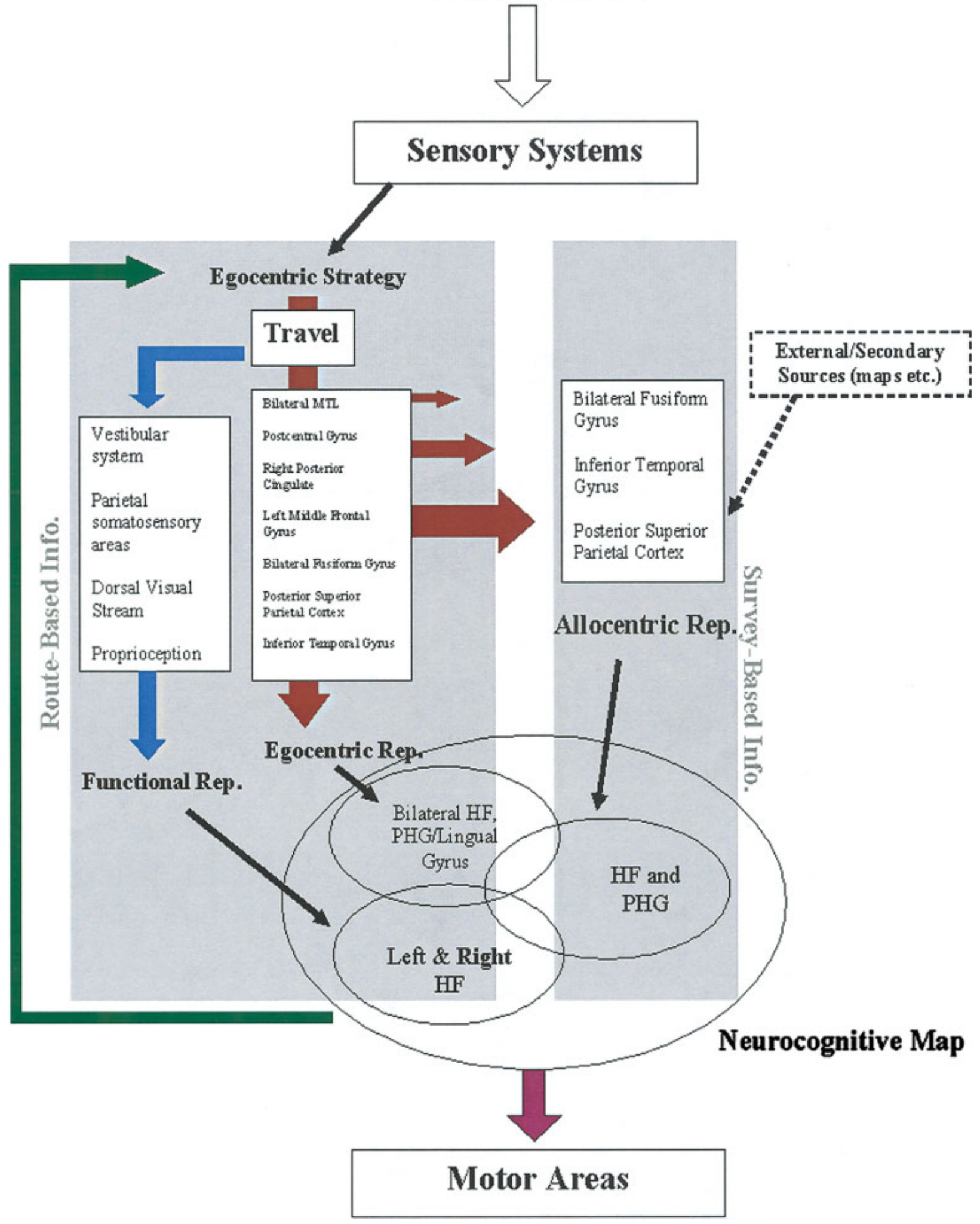

FIGURE 7. Flow diagram of the model indicating brain structures involved in the acquisition/encoding of different forms of spatial representation, and substrates underpinning retrieval from the cognitive map. [Color figure can be viewed in the online issue, which is available at www.interscience.wiley.com.] 
initiate again. Successful navigation and wayfinding will draw upon these three aspects of the neurocognitive map; there is some measure of redundancy between these differing aspects of the map, depending on the complexity of the environment to be navigated. We provide a detailed table of predictions based on the theoretical model presented below (Table 1).

Our proposal-that there is a spatial representation system that consists of multiple parallel processes (pertaining to visual and motoric information) resulting in an integrated modelbears similarities to certain influential models within the animal literature, particularly O'Keefe and Nadel's locale and taxon representations (1978), Jacobs and Schenk's bearing, sketch, and integrated maps (2003) and Whishaw's (1998) conception of path integration. The present model may therefore be viewed as an attempt to bridge the divide between the animal and human literatures by suggesting a system that draws upon important models in the animal literature while also being based on the substantial body of human experimental and lesion data. Our model goes further, however, by specifying a detailed set of human brain structures associated with each aspect of the model as well as providing a clear set of predictions which allow the model to be tested empirically in populations of healthy and brain-damaged humans.

Anatomically, the three spatial constructs will draw upon distinct brain structures to differing extents (Fig. 7). Cortical and subcortical areas involved in each of the processes described in the model will correspond to the areas identified in previous studies examining the "encoding" of spatial environments, while areas associated with the "retrieval" of spatial knowledge should be the cortical loci of each of the three overlapping spatial constructs posited in the model. Therefore, under normal circumstances, the egocentric strategy will use mainly visual information, but in the absence of visual cues, auditory, tactile or even olfactory information may be employed, although in these cases the resulting egocentric model will be of inferior resolution. In addition to the sensory and association areas subserving these modalities, this processing system engages $\mathrm{HF}$ and parietal cortex (Aguirre et al., 1998). The parallel process of path integration, which is also triggered by travel through the environment, derives from the vestibular system as well as parietal somatosensory and sensorimotor areas involved in proprioception (Astur et al., 2002). The resulting functional representation will, we predict, be reliant on a subset of MTL areas which are specialized for the processing of vestibulomotor information, specifically the right and left HF proper, with a possible emphasis on right HF (Astur et al., 2002, Fig. 3; Worsley et al., 2001, Fig. 4; Maguire et al., 1998; Ghaem et al., 1997). The development of the allocentric construct will involve many of the same areas responsible for the egocentric strategy, as this model is derived as a byproduct of the developing egocentric map. Shelton and Gabrieli (2002) (Fig. 2) identified some of these areas as the fusiform gyrus, ITG, and superior parietal cortex. The resulting allocentric map will draw heavily on the parahippocampal gyrus/lingual gyrus (Aguirre et al., 1998; King et al., 2002). The activation of the lingual gyrus points to a linguistic function in spatial representation that has been largely ignored in the past; this issue is addressed below.
This model proposes that the nonallocentric representation of the environment, previously conceptualized as "the egocentric representation," should be divided into two separable but overlapping spatial constructs, an egocentric representation, which contains mainly visual, viewpoint-dependent information (like a series of snapshots), and a functional representation, which contains primarily nonvisual knowledge crucial for successful wayfinding (vestibular, proprioceptive etc.) along with minor visual inputs such as optic flow. We contend that previous studies which have examined egocentric representation (and/or the neural correlates thereof) have, in fact, confounded egocentric and functional variables to greater or lesser extents, depending on the particular design. Closer examination of the nature of information investigated in each study allows the two models, and their substrates, to be teased apart. For example, Maguire et al. (1998) proposed that the right HF was specialized to point continuously toward the goal direction. This capacity would appear to draw more heavily on the functional representation than the egocentric "snapshot" model, and therefore the right hippocampal area may be a strong candidate area for this functional model. By contrast, Maguire et al. (1996) reported topographical impairment in left and right hippocampectomy patients who had been exposed to an environment by videotape presentation of a route through the city (which is therefore largely devoid of path integration-type information; Fig. 5A). Similarly, Astur et al.'s (2002) observation of the inability of hippocampectomy patients to combine optokinetic and vestibular information further supports this proposal (see also Ghaem et al., 1997). Also, data from our own laboratory lend further support to the model. In a study featuring the largest sample of hippocampectomy patients to date (Mangaoang et al., 2004), a clear functional dissociation was found: both left and right hippocampectomy patients were impaired on egocentricand allocentric-based tasks (landmark recognition and location, virtual map tasks, landmark pointing) but were unimpaired on a linear navigation task which was heavily reliant on vestibulomotor and proprioceptive information of the sort represented in our functional construct; Mangaoang et al. (2004) also suggests a linguistic role for left hippocampus, given the large emphasis on language for the encoding of routes and landmarks. Further, this finding represents a double dissociation with the data of Nangle (1998), who observed the opposite pattern with a group of parietal-damaged patients who displayed impaired linear navigation but normal landmark- and map-based performance.

While several anatomical structures beyond HF are proposed as the substrates of each process/construct, some areas will necessarily form the primary input sources for each type of representation. As such, we propose that the functional spatial construct will draw primarily upon vestibular and proprioceptive information, with lesser inputs from visual and somatosensory areas. The egocentric construct will be based largely upon distal landmark information derived from activity in fusiform, inferior temporal and posterior parietal areas, with a subset of these areas (most likely fusiform gyrus) responsible for the formation of the allocentric strategy. While this prioritization of structures should allow for more specific predictions to be made regarding imaging and lesion studies, it must be noted that such prioriti- 
zation is difficult because there appear to be multiple redundant systems present within each processing pathway, allowing for substantial compensation of function if one of the inputs should be damaged. In this way, the negative effects of a vestibular system lesion, for example, may go unnoticed (or be "behaviorally silent") (Dickinson, 1980) due to the other inputs relating to path integration (proprioception, visual, somatosensory).

Sufficient methodologies and paradigms have already been developed to allow this model to be tested in the future (for a detailed discussion of these paradigms, see the section, Hippocampal Formation and Spatial Representation), in particular the use of passive and blind navigation studies. Gaunet et al. (2001), for example, compared active, passive and snapshot exploration of a VR city. These three conditions perfectly tease apart the different aspects of the model: active exploration provides a functional model of the city complete with topological knowledge; passive exploration results in a functional model of angles, directions, distances etc. but without any topographic information; snapshot exploration provides only the type of visually-based knowledge available to the egocentric representation. The resulting cognitive map for the snapshot condition would be predicted to be impoverished relative to the other two conditions: this prediction was supported by the data (no difference was found between the two "functional" conditions, possibly because topological information was not tested by the DV used in the study, reproduction of path shape). Furthermore, reexamination of the existing literature with this model in mind suggests that the possible locus of this proposed "functional representation" may be the right and/or left HF proper, as compared with the parahippocampal gyrus (PHG) which may play a larger role in egocentric and allocentric representations.

\section{Predictions Based on the Model}

Our model allows for clear and testable predictions to be made in terms of behavioral performance and anatomical activations under differing experimental conditions. Many of these predictions may be easily tested using simple experimental designs, and using tasks and paradigms that are well established (see the section, Hippocampal Formation and Spatial Representation). We now describe some key predictions that could be tested to falsify the model. Table 1 presents these predictions in summary format.

\section{Behavioral performance}

The model's division of route-based information into two processing streams allows for the formulation of specific behavioral predictions based on the removal or disruption of either processing pathway (Fig. 6). Removal of egocentric spatial information (e.g., by blindfolding participants) will result in an impoverished egocentric representation within the neurocognitive map, while impairing the path integration system (either by passive actual or VR navigation, thereby preventing proprioceptive feedback, or through vestibular disruption by caloric stimulation) will give rise to a less sophisticated functional rep- resentation. (Caloric stimulation is a test which uses differences in temperature to induce dizziness or vertigo. Cold water is injected into the ear canal with a bulb syringe resulting in nystagmus and disorientation.) As a result, performance measures that are heavily reliant on the egocentric model will be impaired in the former condition, while tasks requiring translational knowledge (such as blind navigation) will be disrupted in the latter. Thus, map drawing should be impaired when participants navigate blindfolded, while blindfolded navigation will be disrupted when participants are passively propelled through an environment.

Furthermore, the allocentric representation can be investigated with the use of secondary sources such as maps. In situations where the neurocognitive map develops as a result of such a secondary source (i.e., no actual travel through the environment takes place), the model would predict poorer performance than cases where the representation was derived from purely egocentric information (viewing one or more static images of the environment) or normal path integration. Data from our laboratory support this prediction; poorest performance in blind navigation was found when the neurocognitive map was based on allocentric information, while the greatest accuracy was observed for a path integration group (O'Riordan et al., 2005). Also, in conditions where a secondary source is presented which is erroneous or inaccurate, participants will return to a reliance on route-based information in order to override the faulty allocentric spatial construct in favor of a new, accurate model.

\section{Anatomical specificity}

Experimental tasks which draw upon differing aspects of the neurocognitive map are predicted to elicit increased activity in the corresponding anatomical areas (as shown in Fig. 7), while damage to these regions is predicted to result in impairments on tasks dependent on the associated function. Functional imaging will allow the anatomical substrates of the model's processing stages to be verified. The first clear prediction is for a double dissociation between areas involved in the encoding and subsequent retrieval of each type of representation (Fig. 7). It can also be predicted that the encoding of functional information will activate areas such as the vestibular system, parietal somatosensory and proprioceptive areas, and the dorsal visual stream as well as MTL, whereas retrieval of such functional knowledge will activate bilateral $\mathrm{HF}$, with greater activation in the right hemisphere. In contrast, acquisition of the egocentric representation (though VR or presentation of a series of static views) will activate a collection of areas including MTL bilaterally, postcentral gyrus, posterior superior parietal cortex, fusiform gyrus and ITG. Retrieval of information from the egocentric model will then engage bilateral $\mathrm{HF}$ and the parahippocampal gyrus/lingual gyrus. Finally, when the allocentric representation is generated under normal conditions (i.e., due to physical travel), bilateral fusiform gyrus, ITG, and posterior superior parietal cortex will be activated (as shown by Shelton and Gabrieli, 2002). However, when the representation is 
derived from artificial means such as a map, these areas will likely not be activated, as they are a subset of the egocentric acquisition network. Recall of allocentric representations (induced, for example, by drawing overhead maps of the environment) will result in activation of $\mathrm{HF}$ and parahippocampal gyrus. For future studies, tasks can now be designed specifically to separate aspects of the model's different features and elucidate the anatomical bases of the proposed processes.

In addition to functional imaging, lesion studies will further reveal the operation of the spatial representation system. Patients with damage to $\mathrm{HF}$ or parietal cortex have already been shown to be impaired on a variety of spatial memory and navigation tasks, but recent data has pointed toward an anatomical dissociation of function. Mangaoang et al. (2004) demonstrated intact acquisition of a linear navigation task in a large group of hippocampectomy patients (left and right hemisphere), while they were impaired on egocentric and allocentric tasks. The opposite pattern was shown in a group of parietal patients using the same tasks (Nangle, 1998), suggesting the importance of HF for egocentric and allocentric, but not functional, acquisition. Future studies with patients who have focal lesions of the different areas suggested in the model, and utilizing the experimental designs mentioned above, will help examine the accuracy of the anatomical areas claimed to be involved.

\section{Functional connectivity}

Another strong prediction is that there will be an increase in functional connectivity between certain specific areas during the acquisition of each of the spatial representations within the neurocognitive map. As such, we predict increases in connectivity between bilateral $\mathrm{HF}$ and the areas involved in the path integration system during the encoding of the functional representation of the environment. Similarly, egocentric encoding areas including ITG, fusiform gyrus and middle frontal gyrus should show increased connectivity with bilateral $\mathrm{HF}$ and PHG. Finally, allocentric acquisition areas (bilateral fusiform gyrus, ITG and posterior parietal) will show increased connectivity with HF and PHG. However, if the allocentric model is derived from a secondary source, no such connectivity changes will be seen. Diffusion tensor imaging (DTI) carried out before and after spatial learning of each type should reveal these changes in functional connectivity between areas.

\section{Goal set}

A common element in the notion of spatial navigation is the assumption that the organism knows where they are going, and/ or why they are going there. The model presented does not specifically address this issue of "goal set," but we acknowledge that normal wayfinding will involve decision processes at key landmarks or junctions, and multiple possible routes to a goal destination. We propose that this goal set operation is likely to be subserved by prefrontal areas. In many navigation experiments, participants must follow and remember a single route that does not allow for alternative routes through the environment. We propose, therefore, that in a VR paradigm, participants navigate via a route of their own choosing through an environment with multiple, equally effective routes to a goal destination. We predict that this will cause activation of prefrontal areas compared to a similar task in which no alternate routes were available.

\section{Parallel pathways}

The model suggests that there is activity in parallel pathways during the early encoding phase of the development of the neurocognitive map. Disruption or elimination of one pathway at this stage will leave the other pathway unimpaired, and vice versa. The egocentric pathway can be easily disrupted by removing visual input, while the path integration system can be hampered by vestibular disruption through caloric stimulation or by spinning the participant. In each case, the spatial construct should not be affected by disruption of the other pathway. Further, as the allocentric spatial construct is derived primarily from the egocentric strategy, disrupting this pathway will result in a less developed allocentric representation than would disruption of the path integration system. Thus reducing the amount of visual information available will lead to poorer performance on a map-drawing task.

While the early stages of the system involve parallel pathways, the later stages require the interaction of spatial constructs and the brain regions that subserve them. Imaging studies should allow anatomical evidence for such interactions between brain areas to be revealed, and the relative contributions of these areas to each type of spatial construct to be elucidated.

\section{CONCLUSION}

The model presented is a neurocognitive account of the acquisition and maintenance of an internal representation of the external environment, detailing functional and anatomical dissociations between processing strategies and mental models. It explains anomalies in the existing literature and is supported by behavioral and imaging data in normals, as well as performance data in HF-lesioned patients. In addition, the model gives rise to a collection of clear and testable theoretical, behavioral and anatomical predictions that allow for falsification. Furthermore, these hypotheses may be easily tested using existing technologies (e.g., functional imaging) and well-established experimental paradigms and approaches.

\section{Acknowledgments}

Special thanks to Sid Weiner (CNRS-Collège de France, Paris) for many useful comments.

\section{REFERENCES}

Aguirre GK, D’Esposito M. 1997. Environmental knowledge is subserved by separable dorsal/ventral neural areas. J Neurosci 26:265-272. 
Aguirre GK, D’Esposito M. 1999. Topographical disorientation: a synthesis and taxonomy. Brain 122:1613-1628.

Aguirre GK, Detre JA, Alsop DC, D’Esposito M. 1996. The parahippocampus subserves topographic learning in man. Cereb Cortex $16: 823-829$.

Aguirre GK, Zarahn E, D’Esposito M. 1998. An area within human ventral cortex sensitive to "Building" stimuli: evidence and implications. Neuron 21:373-383.

Astur RS, Oritz ML, Sutherland RJ. 1998. A characterization of performance by men and women in a virtual Morris water task: a large and reliable sex difference. Behav Brain Res 93:185-190.

Astur RS, Taylor LB, Mamelak AN, Philpott L, Sutherland RJ. 2002. Humans with hippocampus damage display severe spatial memory impairments in a virtual Morris water task. Behav Brain Res 132: 77-84.

Beritoff JS. 1965. Neural mechanisms of higher vertebrate behaviour. New York: Little, Brown.

Berthoz A. 1997. Le sens du mouvement. Paris: Odile Jacob.

Berthoz A. 1999. Hippocampal and parietal contribution to topokinetic and topographic memory. In: Burgess N, Jeffery KJ, O'Keefe $\mathrm{J}$, editors. The hippocampal and parietal foundations of spatial cognition. Oxford: Oxford University Press. p 381-403.

Berthoz A, Israel I, Georges-François P, Grasso R, Tsuzuki T. 1995. Spatial memory of body linear displacement: what is being stored? Science 269:95-8.

Bliss TVP, Lømo T. 1978. Long-lasting potentiation of synaptic transmission in the dentate area of the anaesthetized rabbit following stimulation of the perforant path. J Physiol 232:331-356.

Bloch H, Morange F. 1997. Organising gestures in external space: orienting and reaching. In: Foreman N, Gillett R, editors. Handbook of spatial research paradigms and methodologies. Vol 2. Hove: Psychology Press.

Burgess N, O'Keefe J. 1996. Neuronal computations underlying the firing of place cells and their role in navigation. Hippocampus 6: 749-762.

Burgess N, Maguire EA, O’Keefe J. 2002. The human hippocampus and spatial and episodic memory. Neuron 35:625-641.

Canning KJ, Leung LS. 1997. Lateral-entorhinal, perirhinal amygdalaentorhinal transition projections to hippocampal CA1 and dentate gyrus in the rat: a current source density study. Hippocampus 7 : 643-655.

Christou CG, Bulthoff HH. 1999. The perception of spatial layout in a virtual world. Technical report. Tubingen, Germany: Max Planck Institute for Biological Cybernetics.

Cohen NJ, Eichenbaum H. 1993. Memory, amnesia, and the hippocampal system. Cambridge, MA: MIT Press.

Couclelis H, Golledge RG, Gale N, Tobler W. 1987. Exploring the anchor-point hypothesis of spatial cognition. J Environ Psychol 5: 99-122.

Daniel M-P, Denis M. 1998. Spatial descriptions as navigational aids: a cognitive analysis of route directions. Kognitionwissenschaft 7: 45-52.

Dash PK, Herbert AE, Runyan AD. 2004. A unified theory for systems and cellular memory consolidation. Brain Res Rev 45:30-37.

Dickinson A. 1980. Contemporary animal learning theory. Cambridge, UK: Cambridge University Press.

Diwadkar VA, McNamara TP. 1997. Viewpoint dependence in scene recognition. Psychol Sci 8:302-307.

Duvernoy HM. 1991. The human brain: surface, three-dimensional sectional anatomy, and MRI. Vienna: Springer.

Easton RD, Sholl MJ. 1995. Object-array structure, frames of reference, and retrieval of spatial knowledge. J Exp Psychol Learn Mem Cogn 21:483-500.

Epstein R, Kanwisher N. 1998. A cortical representation of the local visual environment. Nature 392:599-601.

Etienne AS. 1992. Navigation of a small mammal by dead reckoning and local cues. Curr Dir Psychol Sci 1:48-52.
Feigenbaum JD, Rolls ET. 1991. Allocentric and egocentric information processing in the hippocampal formation of the behaving primate. Psychobiology 19:21-40.

Foreman N, Gillett R. 1997. Handbook of spatial res paradigms and methodologies. vol. 1. Spatial cognition in the child and adult. Hove: Psychology Press.

Garling T, Golledge RG. 2000. Cognitive mapping and spatial decision-making. In: Kitchin R, Freundschuh S, editors. Cognitive mapping: past, present and future. London: Routledge.

Gaunet F, Vidal M., Kemeny A, Berthoz A. 2001. Active, passive and snapshot exploration in a virtual environment: influence on scene memory, reorientation and path memory. Cogn Brain Res 11:409-420.

Gavrilov VV, Wiener SI, Berthoz A. 1995. Enhanced hippocampal theta EEG during whole body rotations in awake restrained rats. Neurosci Lett 197:239-241.

Ghaem O, Mellet E, Crivello F, Tzourio N, Mazoyer B, Berthoz A, Dennis M. 1997. Mental navigation along memorized routes activates the hippocampus, precuneus and insula. NeuroReport 8:739-744.

Gibson JJ. 1950. The perception of the visual world. Boston: Houghton Mifflin.

Glauser S, Amorim MA, Vitte E, Berthoz A. 1994. Goal directed linear locomotion in normal and labryinthine defective subjects. Exp Brain Res 98:323-335.

Golledge RG. 1999. Human wayfinding and cognitive maps. In: Golledge $\mathrm{R}$, editor. Wayfinding behaviour. Baltimore, MA: Johns Hopkins University Press. p 5-45.

Healy S. 1998. Spatial representation in animals. Oxford: Oxford University Press.

Heft H. 1996. The ecological approach to navigation: a Gibsonian perspective. In: Portugali $\mathrm{H}$, editor. The construction of cognitive maps. Dordrecht, the Netherlands: Kluwer Academic. p 105-132.

Held R, Durlach N. 1992. Telepresence. Presence 1:109-112.

Ito S, Odahara S, Hirano M. 1992. Cristospinal reflex in circular walking. Acta Otolaryngol (Stockh) 112:170-173.

Ito S, Odahara S, Hiraki M, Idate M. 1995. Evaluation of imbalance of the vestibulo-spinal reflex by "the circular walking test." Acta Otalaryngol (Stockh) S519:124-126.

Jacobs LF. 1995. The ecology of spatial cognition: adaptive patterns of hippocampal size and space use in wild rodents. In: Alleva E, Fasolo A, Lipp H-P, Nadel L, editors. Studies of the brain in naturalistic settings. Dordrecht, the Netherlands: Kluwer Academic. p 301-322.

Jacobs LF, Schenk F. 2003. Unpacking the cognitive map: the parallel map theory of hippocampal function. Psychol Rev 110:285-315.

Kamil AC. 1994. A synthetic approach to the study of animal intelligence. In: Real LA, editor. Behavioral mechanisms in evolutionary ecology. Chicago: University of Chicago Press. p 11-45.

King JA, Burgess N., Hartley T, Vargha-Khadem F, O’Keefe J. 2002. Human hippocampus and viewpoint dependence in spatial memory. Hippocampus 12:811-820.

Lavenex P, Amaral DG. 2000. Hippocampal-neocortical interaction: a hierarchy of associativity. Hippocampus 10:420-430.

Levine DN, Warach J, Farah MJ. 1985. Two visual systems in mental imagery: dissociation of "what" and "where" in imagery disorders due to bilateral posterior cerebral lesions. Neurology 35:10101018.

Leung LS, Roth L, Canning KJ. 1995. Entorhinal inputs to hippocampal CA1 and dentate gyrus in the rat: a current source density study. J Neurophysiol 73:2392-2403.

Lobel E, le Bihan D, Leroy-Willeg A, Berthoz A. 1996. Searching for the vestibular cortex with functional MRI, (Abstract). NeuroImage 3: S351.

Loomis JM, Klatzky RL, Golledge RG, Cicinelli JG, Pellegrino JW, Fry PA. 1993. Nonvisual navigation by blind and sighted: assessment of path integration ability. J Exp Psychol Gen 122:73-91.

Loomis JM, Klatzky RL, Golledge RG, Philbeck JW. 1999. Human navigation by path integration. In: Golledge RG, Philbeck JW, 
editors. Wayfinding: cognitive mapping and spatial behaviour. Baltimore, MD: Johns Hopkins University Press. p 125-51.

Lopes da Silva FH, Witter MP, Boeijinga PH, Lohman AHM. 1990. Anatomical organization and physiology of the limbic system. Physiol Rev 70:453-511.

MacEachren AM. 1992. Application of environmental learning theory to spatial knowledge acquisition from maps. Ann Assoc Am Geog 82:245-274.

Maguire EA, Frackowiak RSJ, Frith CD. 1997. Recalling routes around London: activation of the right hippocampus in taxi drivers. J Neurosci 17:7103-7110.

Maguire EA, Frith CD, Burgess N, Donnett JG, O'Keefe J. 1998. Knowing where things are: parahippocampal involvement in encoding object locations in virtual large-scale space. J Cogn Neurosci 10:61-76.

Maguire EA, Burgess N, O’Keefe J. 1999. Human spatial navigation: cognitive maps, sexual dimorphism, and neural substrates. Curr Opin Neurobiol 9:171-177.

Maguire EA, Burke T, Phillips J, Staunton H. 1996. Topographical disorientation following unilateral temporal lobe lesions in humans. Neuropsychologia 34:132-136.

Mangaoang MA, McMackin D, Quigley J, O’Mara SM. 2004. The effects of left and right selective amygdalohippocampectomy on everyday memory, discourse production and spatial representation. FENS Forum Abs 2:A124.19.

Markus EJ, Barnes CA, McNaughton BL, Gladden VL, Skaggs WE. 1994. Spatial information content and reliability of hippocampal CA1 neurons: effects of visual input. Hippocampus 4:410-421.

Mayes AR, Montaldi D. 1999. The neuroimaging of long-term memory encoding processes. Memory 7:613-659.

McNaughton BL, Barnes CA, Gerrard JL, Gothard K, Jung MW, Knierim JJ, Kudrimoti H, Qin Y, Skaggs WE, Suster M, Weaver KL. 1996. Deciphering the hippocampal polyglot: the hippocampus as a path integration system. J Exp Biol 199:173-85.

Mellet E, Bricogne S, Crivello B, Mazoyer MD, Tzourio-Mazoyer N. 2002. Neural bases of mental scanning of a topographic representation built from a text. Cereb Cortex 12:1322-1330.

Millar S. 1994. Understanding and representing space: theory and evidence from studies with blind and sighted children. Oxford: Clarendon Press.

Mittelstaedt H, Glasauer S. 1991. Idiothetic navigation in gerbils and humans. Zool J Physiol 95:427-435.

Mittelstaedt ML, Mittelstaedt H. 2001. Idiothetic navigation in humans: estimation of path length. Exp Brain Res 139:318-332.

Mumby DG, Astur RS, Weisend MP, Sutherland RJ. 1999. Retrograde amnesia and selective damage to the hippocampal formation: memory for places and object discriminations. Behav Brain Res 106:97107.

Naber PA, Caballero-Bleda M, Jorritsma-Byham B, Witter MP. 1997. Parallel input to the hippocampal memory system through periand postrhinal cortices. NeuroReport 8:2617-2621.

Naber PA, Witter MP, Lopes de Silva FH. 1999. Perirhinal cortex input to the hippocampus in the rat: evidence for parallel pathways, both direct and indirect. A combined physiological and anatomical study. Eur J Neurosci 11:4119-4133.

Nangle N. 1998. An investigation of spatial orientation in right parietal stroke patients. Unpublished doctoral thesis. University of Dublin, Trinity College, Dublin, Ireland.

Nakamura K, Kawashima R, Sato N, Nakamura A, Sugiura M, Kato T, Hatano K, Ito K, Fukuda H, Schormann T, Zilles K. 2000. Functional delineation of the human occipito-temporal areas related to face and scene processing. A PET study. Brain 123:1903-1912.

O'Keefe J, Dostrovsky J. 1971. The hippocampus as a spatial map: preliminary evidence from unit activity in the freely moving rat. Brain Res 34:171-175.

O'Keefe J, Nadel L. 1978. The hippocampus as a cognitive map. Oxford: Clarendon Press.
O’Mara SM. 1995. Spatially selective firing properties of hippocampal formation neurons in rodents and primates. Prog Neurobiol 45: 253-274.

O'Mara SM, Rolls ET, Berthoz A, Kesner RP. 1994. Neurons responding to whole-body motion in the primate hippocampus. J Neurosci 14:6511-6523.

O’Riordan K, Roche RAP, Mangaoang MA, O’Mara SM. 2005. Prior spatial navigation facilitates nonvisual wayfinding in humans. Perception (submitted).

Overman WH, Pate BJ, Moore K, Peuster A. 1996. Ontogeny of place learning in children as measured in the radial arm maze, Morris search task, and open field task. Behav Neurosci 110:1205-1228.

Peruch P, Gaunet F, Thinus-Blanc C, Loomis J. 2000. Understanding and learning virtual spaces. In: Kitchin R, Freundschuh S, editors. Cognitive mapping: past, present and future. London: Routledge.

Potegal M. 1982. Vestibular and neostriatal contribution to spatial orientation. In: Potegal M, editor. Spatial abilities. Development and physiological foundations. San Diego, CA: Academic Press. p 36187.

Redish AD. 1999. Beyond the cognitive map. Cambridge, MA: MIT Press.

Rieser JJ. 1989. Access to knowledge of spatial structure at novel points of observation. J Exp Psychol Learning Mem Cogn 15: $1157-1165$.

Rolls ET. 1996. A theory of hippocampal function in memory. Hippocampus 6:601-620.

Rolls ET, O’Mara SM. 1995. View-responsive neurons in the primate hippocampal complex. Hippocampus 5:409-424.

Rolls ET, Baylis GC, Hasselmo ME, Nalwa V. 1989a. The effect of learning on the face selective responses of neurons in the cortex in the superior temporal sulcus of the monkey. Exp Brain Res 76: 153-164.

Rolls ET, Miyashita Y, Cahusac P, Kesner RP, Niki HD, Feigenbaum JD, Bach L. 1989b. Hippocampal neurons in the monkey with activity related to the place where a stimulus is shown. J Neurosci 9:153-164.

Sandstrom NJ, Kaufman J, Huettel SA, 1998. Males and females use different distal cues in a virtual environment navigation task. Cogn Brain Res 6:351-360.

Schenk F. 1998. The Morris water maze is not a maze. In: Foreman N, Gillett R, editors. Handbook of spatial research paradigms and methodologies. Vol 2. Clinical and comparative studies. Hove: Psychology Press. p 145-188.

Scoville WB, Milner B. 1957. Loss of recent memory after bilateral hippocampal lesions. J Neurol Neurosurg Psychiatry 20:11-21.

Sharp PE, Blair HT, Etkin D, Tzanetos DB. 1995. Influences of vestibular and visual motion information on the spatial firing patterns of hippocampal place cells. J Neurosci 15:173-189.

Shelton AL, Gabrieli JDE. 2002. Neural correlates of encoding space from route and survey perspectives. J Neurosci 22:2711-2717.

Shettleworth SJ. 1998. Cognition, evolution, and behavior. Oxford: Oxford University Press.

Sholl MJ. 1996. From visual information to cognitive maps. In: Portugali J, editor. The construction of cognitive maps. Dordrecht, the Netherlands: Kluwer Academic. p 157-186.

Siegel AW, White SH. 1975. The development of spatial representations of large-scale environments. In: Reese HW, editor. Advances in child development and behaviour. Vol 10. New York: Academic Press. p 9-55.

Smith PF. 1997. Vestibular-hippocampal interactions. Hippocampus $7: 465-471$.

Spiers HJ, Burgess N, Hartley T, Vargha-Khadem F, O’Keefe J. 2001a. Bilateral hippocampal pathology impairs topographical and episodic memory but not visual pattern matching. Hippocampus 11: $715-725$

Spiers HJ, Burgess N, Maguire EA, Baxendale SA, Hartley T, Thompson PJ, O'Keefe J. 2001b. Unilateral temporal lobectomy patients 
show lateralized topographical and episodic memory deficits in a virtual town. Brain 124:2476-2489.

Spiers HJ, Maguire EA, Burgess N. 2001c. Hippocampal amnesia. Neurocase 7:357-382.

Squire LR. 1992. Memory and the hippocampus: a synthesis from findings with rats, monkeys, and humans. Psychol Rev 99:195-231.

Suzuki WA, Amaral DG. 1994a. Perirhinal and parahippocampal cortices of the macaque monkey: cortical afferents. J Comp Neurol 350:497-533.

Suzuki WA, Amaral DG. 1994b. Topographic organization of the reciprocal connections between monkey entorhinal cortex and the perirhinal and parahippocampal cortices. J Neurosci 14:18561877.

Takei Y, Grasso R, Amorim M-A, Berthoz A. 1997. Circular trajectory formation during blind locomotion: a test for path integration and motor memory. Exp Brain Res 115:361-368.

Takei Y, Grasso R, Berthoz A. 1996. Quantitative analysis of human walking trajectory on a circular path in darkness. Brain Res Bull 40:491-496.

Taube JS. 1995. Head direction cells recorded in the anterior thalamic nuclei of freely moving rats. J Neurosci 10:420-435.

Telford L, Howard IP, Ohmi M. 1995. Tilt perception during dynamic linear acceleration. Exp Brain Res 104:502-510.

Tellevik JM. 1992. Influence of spatial exploration patterns by blindfolded sighted persons. J Vis Impair Blindness 86:221-224.

Thinus-Blanc C, Gaunet F. 1999. Spatial processing in animals and humans. In: Golledge RG, editor. Wayfinding behaviour. Baltimore, MD: Johns Hopkins University Press.

Thinus-Blanc C, Gaunet F. 1997. Representation of space in blind persons: vision as a spatial sense? J Visual Impair Blindness 86: 221-224.

Thinus-Blanc C, Save E, Poucet B. 1998. Animal spatial cognition and exploration. In: Foreman N, Gillett R, editors. Handbook of spatial res paradigms and methodologies. Vol 2. Clinical and comparative studies. Hove: Psychology Press. p 59-86.

Thomson J. 1983. Is continuous visual monitoring necessary in visually guided locomotion? J Exp Psychol 9:427-443.

Thorndyke PW, Hayes-Roth B. 1982. Differences in spatial knowledge acquired from maps and navigation. Cogn Psychol 14:560-589.

Tolman EC. 1948. Cognitive maps in rats and men. Psychol Rev 55:189-208.
Tversky B. 1991. Spatial mental models. In: Bower GH, editor. The psychology of learning and motivation. San Diego: Academic Press. p $109-145$.

Tversky B. 2000. Levels and structure of spatial knowledge. In: Kitchin R, Freundschuh S, editors. Cognitive mapping: past, present and future. London: Routledge. p 24-43.

Ungar S. 2000. Cognitive mapping without visual experience. In: Kitchin R, Freundschuh S, editors. Cognitive mapping: past, present and future. London: Routledge. p 221-248.

Uttal DH. 1994. Preschoolers' and adults' scale translation and reconstruction of spatial information acquired from maps. Br J Dev Psychol 12:259-275.

Van Hoesen GW. 1995. Anatomy of the medial temporal lobe. Magn Res Imag 13:1047-1055.

Vargha-Khadem F, Gadin DG, Watkins KE, Connelly A, Van Paesschen W, Mishkin M. 1997. Differential effects of early hippocampal pathology on episodic and semantic memory. Science 277:376380.

Wang RF, Simons DJ. 1999. Active and passive scene recognition across views. Cognition 70:191-210.

Whishaw IQ. 1998. Place learning in hippocampal rats and the path integration hypothesis. Neurosci Biobehav Rev 22:209-220.

Whiteley AM, Warrington EK. 1978. Selective impairment of topographical memory; a single case study. J Neurol Neurosurg Psychiatry 41:575-578.

Wiener SI, Korshunov VA, Garcia R, Berthoz A. 1995. Inertial, substratal and landmark cue control of hippocampal CA1 place cell activity. Eur J Neurosci 7:2206-2219.

Witmer BG, Singer MJ. 1994. Measuring presence in virtual environments. ARI Technical Report 1014. Alexandra, VA: US Army Research Institute for the Behavioral and Social Sciences.

Witter MP. 1989. Connectivity of the rat hippocampus. In: ChanPalay V, Kohler C, editors. The hippocampus. New York: Alan R Liss. p 53-69.

Witter MP, Groenewegan HJ. 1992. Organizational principles of hippocampal connections. In: Trimble MR, Bolwig TG, editors. The temporal lobes and the limbic system. Petersfield, UK: Wringstom Biomedical. p 37-60.

Worsley CL, Recce M, Spiers HJ, Marley J, Polkey C, Morris RG. 2001. Path integration following temporal lobectomy in humans. Neuropsychologia 39:452-464. 